\title{
Permutation groups, minimal degrees and quantum computing
}

\author{
Julia Kempe, László Pyber and Aner Shalev \\ To Avinoam Mann, teacher, colleague and friend
}

\begin{abstract}
We study permutation groups of given minimal degree without the classical primitivity assumption. We provide sharp upper bounds on the order of a permutation group $H \leq S_{n}$ of minimal degree $m$ and on the number of its elements of any given support. These results contribute to the foundations of a non-commutative coding theory.

A main application of our results concerns the Hidden Subgroup Problem for $S_{n}$ in quantum computing. We completely characterize the hidden subgroups of $S_{n}$ that can be distinguished from identity with weak Quantum Fourier Sampling, showing that these are exactly the subgroups with bounded minimal degree. This implies that the weak standard method for $S_{n}$ has no advantage whatsoever over classical exhaustive search.
\end{abstract}

Mathematics Subject Classification (2000). 20B35, 20C15, 68Q25, 81P68.

Keywords. Permutation groups, quantum computing, minimal degree, Hidden Subgroup Problem, characters.

\section{Introduction}

Let $S_{n}$ denote the symmetric group on $\{1, \ldots, n\}$. For a permutation $h \in S_{n}$ define its support $\operatorname{supp}(h)$ by

$$
\operatorname{supp}(h)=\{i \in\{1, \ldots, n\}: h(i) \neq i\} .
$$

The minimal degree $m(H)$ of a permutation group $1 \neq H \leq S_{n}$ is defined to be the minimal number of points moved by a non-identity element of $H$. In other words,

$$
m(H)=\min \{|\operatorname{supp}(h)|: 1 \neq h \in H\} .
$$

This notion goes back to the 19th century, and plays an important role in the theory of finite permutation groups since the days of Jordan [16], [17]. Particular attention was given to the minimal degree of primitive permutation groups. Recall that a permutation 
group is called primitive if it is transitive and does not preserve a non-trivial block system. Let $H<S_{n}$ be a primitive permutation group not containing $A_{n}$. Jordan proved that $m(H)$ goes to infinity as $n$ goes to infinity. Babai [1] showed that under the above conditions we actually have that

$$
m(H) \geq \frac{\sqrt{n}-1}{2} .
$$

This result is essentially best possible. However, if we exclude certain primitive groups and use the Classification of Finite Simple Groups (CFSG) (see e.g. [9]), sharper bounds can be obtained. Indeed, it was shown by Liebeck and Saxl in [24] that $m(H) \geq n / 3$ with a given list of exceptions. This lower bound was improved by Guralnick and Magaard in [12] to $n / 2$ (with prescribed exceptions). See also Cameron [4] for the impact of the Classification on the theory of finite permutation groups and primitive groups in particular.

In spite of considerable progress in the study of the minimal degree of primitive groups, much less is known in the non-primitive case. One of the purposes of this paper is to study permutation groups of given minimal degree without assuming primitivity or even transitivity.

A basic question in this field is: how large can a permutation group $H$ of degree $n$ and minimal degree $m$ be? An easy classical upper bound is $|H| \leq n^{n-m+1}$. Indeed, this follows from the fact that a permutation $h \in H$ is uniquely determined by its action on $\{1, \ldots, n-m+1\}$.

Better bounds were given by Liebeck [22], [23] under the assumption that $H$ is transitive. Our first result extends Liebeck's theorem to arbitrary permutation groups.

Theorem A. Let $H \leq S_{n}$ be a permutation group with minimal degree $m=m(H)$.

1) If $m \leq \log _{2} n$, then $|H| \leq n^{10 n / m}$.

2) If $m \geq \log _{2} n$, then $|H| \leq 2^{10 n}$.

Theorem A is essentially best possible. For example, consider the group $H=$ $S_{2 n / m}<S_{n}$ acting on $2 n / m$ blocks of size $m / 2$. Then the minimal degree of $H$ is $m$ and $|H|=(2 n / m)$ ! which is of the form $n^{(2-o(1)) n / m}$ when $m \leq \log _{2} n$. Up to a constant in the exponent, this shows that part (1) of Theorem A is tight.

Note also that if $H \leq S_{n}$ is transitive of minimal degree $m$ and base size $b$, then $b m \geq n$ (see e.g. [5], p. 80), and this implies $|H| \geq 2^{b} \geq 2^{n / m}$.

Subgroups of $S_{n}$ of given minimal degree $m$ can be regarded as non-commutative analogues of linear codes with minimal distance $m$. Recall that in coding theory [26] a fundamental question is: how large can a subspace of $\operatorname{GF}(q)^{n}$ with minimal distance $m$ be? Replacing the Abelian group $\mathrm{GF}(q)^{n}$ by the symmetric group $S_{n}$ we may ask a similar question in this context. Theorem A provides a rather sharp answer. 
Note that any binary linear code inside $\operatorname{GF}(2)^{n / 2}$ can be embedded naturally as a subgroup of $S_{2}^{n / 2}<S_{n}$. Thus classical coding theory provides a rich source of constructions of permutation groups of large minimal degree. In particular the (obvious) Gilbert-Varshamov lower bound ([10] p. 781, remark after Thm. 3.5) applied to linear codes produces exponentially large elementary Abelian permutation groups with large minimal degree, e.g. $m>n / 8$. This demonstrates the tightness of part (2) of Theorem A, even when $m$ is very large.

Another classical question in coding theory is the study of the weight distribution, namely counting elements of weight $k$ in a code with minimal distance $m$. The analogous question for permutation groups is counting the number of elements of support $k$ in a permutation group of minimal degree $m$. Given a permutation group $H \leq S_{n}$ define

$$
H_{k}=\{h \in H:|\operatorname{supp}(h)|=k\},
$$

the subset of elements of support $k$ in $H$. In our second result, which is the most technically demanding, we bound the size of $H_{k}$.

Theorem B. There exists absolute constants $b, \varepsilon>0$ such that if a subgroup $H \leq S_{n}$ has minimal degree $m \geq b$ then

$$
\left|H_{k}\right| \leq n^{-\varepsilon m}\left(\begin{array}{l}
n \\
k
\end{array}\right)^{\frac{1}{2}}(k !)^{\frac{1}{4}} .
$$

The theorem has an interesting consequence for the number of elements of minimal support. If $k=m \leq n^{2 \varepsilon}$ then $(k !)^{1 / 4} \leq n^{\varepsilon m / 2}$ and this implies

$$
\left|H_{m}\right| \leq n^{-\varepsilon m / 2}\left(\begin{array}{l}
n \\
m
\end{array}\right)^{1 / 2} .
$$

This upper bound is essentially tight. To show this we use some results from coding theory and the above embedding of binary codes in $S_{n}$. Consider the well known Goppa code [8] and the estimates for the number of code words of minimal weight [21]. For a binary Goppa code over $\mathrm{GF}(2)^{n / 2}$, in the regime of small $t$ $(t \ll \sqrt{\log n}$ ), the number of code words of minimum weight $2 t+1$ is roughly (up to a constant factor)

$$
\left(\begin{array}{c}
n / 2 \\
2 t+1
\end{array}\right)\left(\frac{n}{2}\right)^{-t} .
$$

Embedding this code into $S_{n}$ as above, we obtain a subgroup $H<S_{n}$ of minimal degree $m=4 t+2$ satisfying

$$
\left|H_{m}\right| \geq c n^{-m / 4}\left(\begin{array}{l}
n \\
m
\end{array}\right)^{\frac{1}{2}}
$$


for some constant $c>0$. This demonstrates the tightness of Theorem B in the regime of small $m$.

A main motivation behind Theorem B, besides the study of weight distributions of non-commutative codes, comes from quantum computing. A central problem in quantum computing is the Hidden Subgroup Problem (HSP), which we state below. Let $G$ be a finite group and $H \leq G$ a subgroup. Given a function $f: G \rightarrow S$ that is constant on (left)-cosets $g H$ of $H$ and takes different values for different cosets, determine a set of generators for $H$. The decision version of this problem is to determine whether there is a non-identity hidden subgroup or not.

Note that given $g \in G$ we have $g \in H$ if and only if $f(g)=f(1)$. Using classical search we may therefore perform membership tests, and once we find a nonidentity element $g \in H$ we may conclude that $H \neq 1$. However, the aim is to decide whether or not $H=1$ in polynomial time, namely after $(\log |G|)^{c}$ steps. Complete enumeration over the elements $g \in G$ is therefore not efficient. The question is whether a quantum computer can solve the HSP efficiently (giving the correct answer in polynomial time with a very high probability).

The Hidden Subgroup Problem plays a central role in quantum computing. The two important quantum algorithms for factoring and discrete log, which significantly improve the known classical algorithms, solve the Abelian version of this problem by the so called standard method of Quantum Fourier Sampling. One of the most important questions is whether the standard method can efficiently solve the nonAbelian HSP, especially for the symmetric group $G=S_{n}$. This latter case in particular would yield a quantum algorithm for the Graph Isomorphism Problem, for which no efficient classical algorithm is known. For more details on quantum computing, the HSP, and the standard method see Section 2.

To state our main quantum-theoretic application in a precise mathematical way we need some notation. Given a finite group $G$ let $\widehat{G}$ denote the set of (complex) irreducible representations of $G$ (up to equivalence). For $\rho \in \widehat{G}$ let $d_{\rho}$ denote its dimension and $\chi_{\rho}$ its character.

Given a subgroup $H \leq G$, define

$$
D_{H}=\frac{1}{|G|} \sum_{\rho \in \widehat{G}} d_{\rho}\left|\sum_{h \in H, h \neq 1} \chi_{\rho}(h)\right| .
$$

Roughly speaking, $D_{H}$ measures the $L_{1}$-distance between a (non-commutative) Fourier transform of the characteristic function of $H$ and that of the characteristic function of the identity.

We say that a subgroup $H \leq G$ is distinguishable if

$$
D_{H} \geq(\log |G|)^{-c}
$$

for some constant $c$. Of course this is an asymptotic notion, where we think of $G$ as ranging over an infinite family of groups, whereas the constant $c$ does not depend 
on $G$. Here we focus on the case $G=S_{n}$, where distinguishability is equivalent to $D_{H} \geq n^{-c}$. Distinguishable subgroups $H$ are those which can be distinguished from 1 using the so called weak standard method (see the next section for more details).

The main application of this paper to quantum computing, which relies heavily on Theorem B above, is the following.

Theorem C. Let $H \leq S_{n}$ be a subgroup. If $H$ is distinguishable, then it has a bounded minimal degree. Moreover, if $D_{H} \geq n^{-c}$, then $m(H) \leq g(c)$, where $g(x)=a x+b$ is some fixed linear function.

Thus all subgroups of unbounded minimal degree are indistinguishable, which opens up a huge spectrum of examples and constructions. The only case previously known in the literature of an indistinguishable subgroup of $S_{n}$ is that of a subgroup of order 2 generated by a fixed point free involution or by a product of transpositions of large support [14], [11]. Obviously $m(H)$ is unbounded for such subgroups $H$, so its indistinguishablity is an immediate consequence of the above theorem.

In an extended abstract [18] a subset of the authors of this paper have proved a weaker version of Theorem $C$ (for primitive subgroups and subgroups of polynomial size) and have conjectured that it holds in full generality. In this paper we prove the conjecture.

It is intriguing that much larger subgroups are also indistinguishable. Indeed take $H=S_{2 n / m}<S_{n}$, the subgroup constructed following Theorem A. If $m=m(H)$ tends to infinity arbitrarily slowly, then $H$ is indistinguishable and $|H| \geq(n !)^{\varepsilon(n)}$ where $\varepsilon(n)$ tends to 0 arbitrarily slowly. In particular, the size of indistinguishable subgroups of $S_{n}$ can be super-exponential in $n$.

However, if $\varepsilon>0$ is fixed, and $|H| \geq(n !)^{\varepsilon}$, then it follows from Theorem A that the minimal degree of $H$ is bounded. Enumerating over elements of $S_{n}$ of bounded support (their number is bounded by a polynomial in $n$ ) we deduce that such a subgroup $H$ can be distinguished from 1 using classical search.

It follows from the two paragraphs above that all subgroups $H \leq S_{n}$ of size $\geq N$ can be distinguished from 1 using the weak standard method (together with classical search) if and only if $N \geq(n !)^{\varepsilon}$ where $\varepsilon$ is bounded away from zero.

Theorem $\mathrm{C}$ has rather grave consequences. Indeed, if $H$ is distinguishable then it has an element of bounded support, and this can be detected (as above) after polynomially many membership tests (when we enumerate the permutations in $S_{n}$ according to their support).

Corollary D. Any subgroup $H \leq S_{n}$ which is distinguishable can already be distinguished from 1 using classical search.

Thus Theorem $\mathrm{C}$ provides a complete characterisation of hidden subgroups $H \leq S_{n}$ which can be distinguished from 1 using the weak standard method and classical search: these are precisely the subgroups of bounded minimal degree. 
It is intriguing that the old classical notion of minimal degree, which is central in the theory of finite permutation groups, plays a role in the context of quantum computing. The Classification of Finite Simple Groups (CFSG) is also used in an essential way in some parts of this work.

Some words on the structure of this paper. In Section 2 we provide background on quantum computing, the Hidden Subgroup Problem, and the standard method of Quantum Fourier Sampling. Section 3 deals with arbitrary finite groups $G$ and their subgroups $H$. Using character-theoretic methods we give upper and lower bounds on the $L_{1}$-distance $D_{H}$ introduced above. We then characterize distinguishable subgroups of polylogarithmic size. In Section 4 we focus on the case $G=S_{n}$. We prove there (relying on CFSG and other tools) that any primitive subgroup $H<S_{n}$ not containing $A_{n}$ is indistinguishable. We also show how to deduce Theorem $\mathrm{C}$ from Theorem B. Theorem A is proved in Section 5. Section 6, which is the longest in this paper, is devoted to the proof of Theorem B. This proof applies Theorem A as well as results on primitive groups obtained in Section 4.

\section{Quantum Computing}

In the last decade quantum computation has provided us with powerful tools to solve several problems not known to be classically efficiently solvable, like factoring and discrete $\log$ [35]. This important class of problems and most others where a quantum computer excels more than quadratically with respect to its classical counterpart can be cast into the framework of the Hidden Subgroup Problem (HSP). Let $G$ be a finite group and $H \leq G$ a subgroup. Given a function $f: G \rightarrow S$ that is constant on (left)-cosets $g H$ of $H$ and takes different values for different cosets, determine a set of generators for $H$. The decision version of this problem is to determine whether there is a non-identity hidden subgroup or not.

The reason that quantum computers seem to provide a speed-up for this type of problem is that it is possible to implement the Fourier transform over certain groups efficiently on a quantum computer. This in turn allows to sample the Fourier components efficiently (this technique of Quantum Fourier Sampling is referred to as the "standard method"). In the case of Abelian groups $G$ (appearing in factoring and discrete $\log$ ) the hidden subgroup can be reconstructed with only a polynomial (in $\log |G|$ ) number of queries to the function and a polynomial number of measurements (samplings in the Fourier basis) and postprocessing steps.

We denote states of the vector space $\mathbb{C}[G]$, spanned by the group elements, with a $|\cdot\rangle$, as is standard in quantum computation (see e.g. [30] for more details). 
Definition 1. The Quantum Fourier Transform (QFT) over a group $G$ is the following unitary transformation on $\mathbb{C}[G]$ :

$$
|g\rangle \rightarrow \frac{1}{\sqrt{|G|}} \sum_{\rho, i, j} \sqrt{d_{\rho}} \rho(g)_{i j}|\rho, i, j\rangle
$$

where $\rho$ labels an irreducible representation of $G, d_{\rho}$ is its dimension and $1 \leq i, j \leq d_{\rho}$. The $|\rho, i, j\rangle$ span another basis of $\mathbb{C}[G]$, the so called Fourier basis.

For many non-Abelian groups it is possible to implement the Fourier transform on a quantum computer efficiently (see e.g. [27]), and in particular explicit constructions exist for the symmetric group $S_{n}$ [3].

Addressing the HSP in the non-Abelian case is considered to be one of the most important challenges at present in quantum computing. A positive answer to the question whether quantum computers can efficiently solve the Hidden Subgroup Problem over non-Abelian groups would have several important implications for the solution of problems in NP, which are neither known to be NP-complete nor in P; and which are good candidates for a quantum speed-up. Among the most prominent such problems is Graph Isomorphism, where the group in question is the symmetric group. Hence it is very desirable to get a handle on the power of Quantum Fourier Sampling (QFS) to solve the HSP for general groups.

Definition 2. The standard method of Quantum Fourier Sampling is the following: The state is initialised in a uniform superposition over all group elements; a second register is initialised to $|0\rangle$. Then the function $f$ is applied reversibly over both registers (i.e. $f:|g\rangle|0\rangle \rightarrow|g\rangle|f(g)\rangle$ ). The second register is measured, which puts the first register into the superposition of a (left)-coset of $H$, i.e. in the state $|g H\rangle:=\frac{1}{\sqrt{|H|}} \sum_{h \in H}|g h\rangle$ for some random $g \in G$. Finally the QFT over $G$ is performed, yielding the state

$$
\frac{1}{\sqrt{|G||H|}} \sum_{\rho, i, j} \sqrt{d_{\rho}} \sum_{h \in H} \rho_{i j}(g h)|\rho, i, j\rangle .
$$

A basis measurement now gives $(\rho, i, j)$ with probability

$$
P_{g H}(\rho, i, j)=\frac{d_{\rho}}{|G||H|}\left|\sum_{h \in H} \rho_{i j}(g h)\right|^{2} .
$$

Since we do not know $g$ and $g$ is distributed uniformly, we sample $(\rho, i, j)$ with probability $P_{H}=\frac{1}{|G|} \sum_{g} P_{g H}$. The strong standard method samples both $\rho$ and its entries $i, j$. In the weak standard method only the character $\chi_{\rho}$ is measured (but not the entries $i, j$, which are averaged over). In this case it is not hard to see [14], [11] 
that the probability to sample $\rho$ is independent of the coset of $H$ we happen to land in. Hence the probability to measure $\rho$ in the weak case is

$$
P_{H}(\rho)=\frac{d_{\rho}}{|G|} \sum_{h \in H} \chi_{\rho}(h)
$$

Note that from this expression it is clear that the weak standard method cannot distinguish between conjugate subgroups [14]. Let $\operatorname{Irr}(G)$ be the set of irreducible characters of $G$. Then $P_{H}$ is a distribution on $\operatorname{Irr}(G)$. The strong standard method sometimes provides substantially more information than its weak counterpart, and is indeed necessary to efficiently solve the HSP in the case of groups like the dihedral group [6], [20], [32] and other semidirect product groups [28]. However (see below), for $S_{n}$ it has been shown [11] that for a random basis the additional information provided by the strong method is exponentially small except possibly for very large subgroups.

An even more basic question is which hidden subgroups can be distinguished from the identity via QFS with special attention to the symmetric group. Distinguishing the trivial subgroup $\{e\}$ from a larger subgroup $H$ efficiently using the weak standard method is possible if and only if the $L_{1}$ distance $D_{H}$ between $P_{\{e\}}$ and $P_{H}$ is larger than some inverse polynomial in $\log |G|$. The $L_{1}$ distance (also known as the total variation distance) is given as

$$
D_{H}=\frac{1}{|G|} \sum_{\rho} d_{\rho}\left|\sum_{h \in H, h \neq 1} \chi_{\rho}(h)\right| .
$$

We say that $H$ is distinguishable (using the weak standard method) if $D_{H} \geq$ $(\log |G|)^{-c}$ for some constant $c$, and indistinguishable otherwise.

Several positive results on the power of QFS for the Hidden Subgroup Problem have been obtained previously for groups that are in some ways "close" to Abelian, like some semidirect products of Abelian groups [6], [33], [20], [32], [28], in particular the Dihedral group; Hamiltonian groups [14], groups with small commutator groups [15] and solvable groups of constant exponent and constant length derived series [7]. Often in these cases the irreducible representations are known and can be analysed. For instance the Dihedral group $D_{n}$, the first non-Abelian group to be analysed in this context [6], is "nearly" Abelian in the sense that all of its irreducible representations have degree at most two. Indeed hidden reflections of $D_{n}$ can be distinguished from the identity with only polynomial Quantum Fourier Samplings, similar to the Abelian case (where all irreducible representations are one-dimensional). Note, however, that the computational version of the HSP seems much harder: even though a polynomial number of samples suffice to distinguish hidden reflections information theoretically, no efficient reconstruction procedure is known.

The holy grail of the field is the symmetric group $S_{n}$, which seems much harder to analyse, partly because to this day there is still only partial explicit knowledge about 
its irreducible representations and character values (see e.g. [34]), because most of its subgroups are far from normal (have many conjugate subgroups), because its irreducible representations can have very large dimension (up to almost $(n !)^{1 / 2}$ ) and the number of different irreducible representations is an exponentially small fraction of the size of the group, to name just some of the difficulties. The structure of distinguishable versus indistinguishable subgroups of $S_{n}$ has remained open.

The following results have been obtained for the HSP over the symmetric group: The group $S_{n}$ being non-Abelian, Quantum Fourier Sampling gives a distribution on both the characters and the entries of the corresponding matrix representations. It is shown in [11] that sampling the row index in the strong standard method provides no additional information. They also show that the additional information provided by the strong method in a random basis scales with $\sqrt[3]{|H|^{2} k(G) /|G|}$ where $k(G)$ is the number of conjugacy classes of the group $G$ and $|H|$ the size of the hidden subgroup. Both [14] and [11] show that hidden subgroups of $S_{n}$ of size $|H|=2$, generated by involutions with large support, cannot be distinguished from identity; exactly the task that needs to be solved for Graph Automorphism. Recently, [29] have essentially shown that the strong standard method cannot distinguish the subgroup generated by a fixed point free involution from identity. Moreover, even a generalization of the strong standard method to $O(n \log n)$ instances of Quantum Fourier Sampling does not allow to distinguish the above subgroup from 1 [13]. No results are known for other subgroups of $S_{n}$.

In this work various classical as well as modern parts of the theory of permutation groups are applied for the first time in the context of quantum computing. In our applications to the Hidden Subgroup Problem, we focus on the weak form of the standard method, since the strong form with random choices of basis does not provide any non-negligible additional information for the symmetric group and the subgroups we consider [11]. It remains to be seen whether judicious choices of basis for each irreducible matrix representation can give more information in the case where random choices do not help; but to our knowledge no such examples have been found and in fact recent results of [29] show that in the case of fixed point free involutions no such good basis exists.

Theorem C and Corollary D above provide a complete characterization of subgroups which can be distinguished from 1 using the weak standard method (together with classical exhaustive search). Indeed, these are exactly the subgroups of $S_{n}$ with bounded minimal degree. For instance we cannot distinguish a group generated by a cycle of unbounded length or an involution with unbounded number of transpositions (implying the result in [14], [11]).

This also has implications for the Graph Isomorphism (GI) problem. Recall that to solve GI for two graphs $G_{1}, G_{2}$, it suffices to distinguish a hidden subgroup of the automorphism group $\operatorname{Aut}\left(G_{1} \cup G_{2}\right)$ of the form $H_{1} \times H_{2}$ (not $G_{1} \simeq G_{2}$ ), where $H_{i}=$ $\operatorname{Aut}\left(G_{i}\right)$, from a subgroup of the form $H \cup \sigma H\left(G_{1} \simeq G_{2}\right)$, where $H=H_{1} \times H_{2}$ 
and $\sigma$ maps $G_{1}$ to $G_{2}$. Our results imply that we cannot distinguish each of the two possible cases from identity, and hence (using the triangle inequality) we cannot distinguish them from each other unless $\operatorname{Aut}\left(G_{i}\right)$ contains an element of bounded support. Thus weak QFS provides no advantage here.

\section{Arbitrary groups}

In this section we discuss results for arbitrary finite groups $G$. Our starting point is a general result providing both upper and lower bounds on the total variation distance $D_{H}$ in terms of the same group theoretic data. While the definition of $D_{H}$ involves character degrees and values, which are hard to compute, our bounds below involve sizes of conjugacy classes, and their intersections with the hidden subgroup.

We need some group theoretic notation. For $h \in G$ we let $h^{G}$ denote the conjugacy class of $h$ in $G$. Let $C_{1}, \ldots, C_{k}$ denote the non-identity conjugacy classes of $G$. For an irreducible character $\chi_{\rho} \in \operatorname{Irr}(G)$ we let $\chi_{\rho}\left(C_{i}\right)$ denote the common value of $\chi_{\rho}(x)$ for elements $x \in C_{i}$.

Proposition 1. Let $H<G$. Then

$$
\begin{aligned}
& \text { 1. } \sum_{i=1}^{k}\left|C_{i} \cap H\right|^{2}|H|^{-1}\left|C_{i}\right|^{-1}<D_{H} ; \\
& \text { 2. } D_{H} \leq \sum_{i=1}^{k}\left|C_{i} \cap H\right|\left|C_{i}\right|^{-\frac{1}{2}}=\sum_{1 \neq h \in H}\left|h^{G}\right|^{-1 / 2} .
\end{aligned}
$$

Applying the upper bound with $|H|=2$ gives the result obtained previously by [14] and [11]. No lower bounds seem to exist in the literature. This result has a wide range of applications. For example, it enables us to characterise distinguishable subgroups $H \leq G$ of polylogarithmic order (see Theorem 3 below).

Proof of Proposition 1. For each irreducible representation $\rho$ of $G$ we have

$$
\left|\sum_{h \in H, h \neq 1} \chi_{\rho}(h)\right| \leq \sum_{h \in H, h \neq 1}\left|\chi_{\rho}(h)\right| \leq \sum_{h \in H, h \neq 1} d_{\rho}<|H| d_{\rho} .
$$

Hence $d_{\rho}>|H|^{-1}\left|\sum_{h \in H, h \neq 1} \chi_{\rho}(h)\right|$. Substituting this in (1) we obtain

$$
D_{H}>\frac{1}{|G||H|} \sum_{\rho}\left|\sum_{h \in H, h \neq 1} \chi_{\rho}(h)\right|^{2} .
$$


Note that $\chi_{\rho}(h)=\chi_{\rho}\left(C_{i}\right)$ if $h$ is contained in $H \cap C_{i}$. This yields $\sum_{h \in H, h \neq 1} \chi_{\rho}(h)=$ $\sum_{i=1}^{k}\left|H \cap C_{i}\right| \chi_{\rho}\left(C_{i}\right)$, and so

$$
D_{H}>\frac{1}{|G||H|} \sum_{\rho}\left|\sum_{i=1}^{k}\right| H \cap C_{i}\left|\chi_{\rho}\left(C_{i}\right)\right|^{2}
$$

Now,

$$
\begin{aligned}
& \left|\sum_{i=1}^{k}\right| H \cap C_{i}\left|\chi_{\rho}\left(C_{i}\right)\right|^{2} \\
& \quad=\sum_{i=1}^{k}\left|H \cap C_{i}\right|^{2}\left|\chi_{\rho}\left(C_{i}\right)\right|^{2}+\sum_{i \neq j}\left|H \cap C_{i}\right|\left|H \cap C_{j}\right| \chi_{\rho}\left(C_{i}\right) \bar{\chi}_{\rho}\left(C_{j}\right) .
\end{aligned}
$$

Using the generalised orthogonality relations we observe that

$$
\sum_{\rho} \sum_{i=1}^{k}\left|H \cap C_{i}\right|^{2}\left|\chi_{\rho}\left(C_{i}\right)\right|^{2}=\sum_{i=1}^{k}\left|H \cap C_{i}\right|^{2}|G| /\left|C_{i}\right|,
$$

and

$$
\sum_{\rho} \sum_{i \neq j}\left|H \cap C_{i}\right|\left|H \cap C_{j}\right| \chi_{\rho}\left(C_{i}\right) \bar{\chi}_{\rho}\left(C_{j}\right)=0
$$

It follows that

$$
D_{H}>\frac{1}{|G||H|} \sum_{i=1}^{k}\left|H \cap C_{i}\right|^{2}|G| /\left|C_{i}\right|=\sum_{i=1}^{k}\left|H \cap C_{i}\right|^{2}|H|^{-1}\left|C_{i}\right|^{-1} .
$$

This completes the proof of the lower bound.

To prove the upper bound, write

$$
\begin{aligned}
D_{H}|G|=\sum_{\rho} d_{\rho}\left|\sum_{h \in H, h \neq 1} \chi_{\rho}(h)\right| & \leq \sum_{\rho} d_{\rho} \sum_{h \in H, h \neq 1}\left|\chi_{\rho}(h)\right| \\
& =\sum_{h \in H, h \neq 1} \sum_{\rho} d_{\rho}\left|\chi_{\rho}(h)\right| .
\end{aligned}
$$

Fix $h \in H$ and choose $i$ such that $h \in C_{i}$. Using the Cauchy-Schwarz inequality we obtain

$$
\sum_{\rho} d_{\rho}\left|\chi_{\rho}(h)\right| \leq\left(\sum_{\rho} d_{\rho}^{2}\right)^{1 / 2}\left(\sum_{\rho}\left|\chi_{\rho}(h)\right|^{2}\right)^{1 / 2},
$$


giving (using the orthogonality relations)

$$
\sum_{\rho} d_{\rho}\left|\chi_{\rho}(h)\right| \leq|G|^{1 / 2}\left(|G| /\left|C_{i}\right|\right)^{1 / 2}=|G|\left|C_{i}\right|^{-1 / 2} .
$$

Summing over non-identity elements $h \in H$, and observing that the upper bound above occurs $\left|H \cap C_{i}\right|$ times, we obtain

$$
\sum_{h \in H, h \neq e} \sum_{\rho} d_{\rho}\left|\chi_{\rho}(h)\right| \leq \sum_{i=1}^{k}\left|H \cap C_{i}\right||G|\left|C_{i}\right|^{-1 / 2} .
$$

Combining this with (2) we obtain

$$
D_{H} \leq \sum_{i=1}^{k}\left|H \cap C_{i}\right|\left|C_{i}\right|^{-1 / 2},
$$

as required.

The following is an immediate consequence of Proposition 1.

Corollary 2. Let $C_{\min }$ denote a non-identity conjugacy class of minimal size intersecting $H$ non-trivially. Then we have

$$
|H|^{-1}\left|C_{\min }\right|^{-1}<D_{H} \leq(|H|-1)\left|C_{\min }\right|^{-1 / 2} .
$$

We can now prove the main result of this section, characterising distinguishable subgroups of polylogarithmic order in an arbitrary group $G$.

Theorem 3. Suppose $|H| \leq(\log |G|)^{c}$ for some constant $c$. Then $H$ is distinguishable if and only if $H$ has a non-identity element $h$ such that $\left|h^{G}\right| \leq(\log |G|)^{c^{\prime}}$ for some constant $c^{\prime}$.

Proof. Suppose first that $H$ is distinguishable, namely $D_{H} \geq(\log |G|)^{-b}$ for some constant $b$. Then the upper bound in the above corollary shows that

$$
|H|\left|C_{\min }\right|^{-1 / 2} \geq(\log |G|)^{-b},
$$

so

$$
\left.\left|C_{\min }\right| \leq|H|^{2}(\log |G|)^{2 b}\right) \leq(\log |G|)^{2(b+c)} .
$$

In the other direction, suppose $\left|C_{\min }\right| \leq(\log |G|)^{b}$. Then the lower bound in the corollary above gives

$$
D_{H}>|H|^{-1}(\log |G|)^{-b} \geq(\log |G|)^{-(b+c)} .
$$

The result follows. 


\section{Symmetric groups}

Let us now focus on the case $G=S_{n}$. In this section we first prove some preliminary results related to distinguishability of subgroups of $S_{n}$. Some of these results play a role in the proof of Theorem B. We also deduce Theorem C from Theorem B.

Proposition 4. Let $H \leq S_{n}$ with $|H| \leq n^{c}$ for some constant $c$. Then $H$ is distinguishable if and only if its minimal degree $m(H)$ is bounded.

Proof. Let $g \in S_{n}$ with $\operatorname{supp}(g)=k$. Then it is straightforward to verify that $\left(\begin{array}{l}n \\ k\end{array}\right) \leq\left|g^{S_{n}}\right| \leq n^{k}$. As a consequence we see that a conjugacy class $C$ in $S_{n}$ has polynomial order if and only if it consists of elements of bounded support. This observation, when combined with Theorem 3, completes the proof.

Our next result concerns primitive subgroups. Primitive permutation groups are considered the building blocks of finite permutation groups in general, and were extensively studied over the past 130 years. We note that if $H \leq S_{n}$ is primitive and $H \neq A_{n}, S_{n}$ then Babai showed [1], [2] that $|H| \leq n^{4 \sqrt{n}} \log n$. Using the Classification of Finite Simple Groups the latter bound can be somewhat improved to $|H| \leq 2 n^{\sqrt{n}}$, which is essentially best possible [4]; in particular the order of $H$ can be much more than polynomial, and so Proposition 4 above does not apply.

However, we obtain the following somewhat surprising general result:

Theorem 5. Let $H \neq A_{n}, S_{n}$ be a primitive subgroup of $S_{n}$. Then $H$ is indistinguishable. Moreover, there is an absolute constant $\varepsilon>0$ such that

$$
D_{H} \leq n^{-\varepsilon \sqrt{n}} .
$$

Theorem 5 follows immediately from the two technical lemmas below, which are based on counting elements of given support in permutation groups $H$. Recall that for $H \leq S_{n}$ we set

$$
H_{k}=\{h \in H:|\operatorname{supp}(h)|=k\} .
$$

Lemma 6. Let $H \leq S_{n}$ be a subgroup. Suppose that, for each $k \leq n$, we have

$$
\left|H_{k}\right| \leq n^{(1 / 6-\varepsilon) k} .
$$

where $\varepsilon>0$ is some fixed constant. Then, if $n$ is large enough (given $\varepsilon$ ) we have

$$
D_{H} \leq 2 n^{-\delta m(H)},
$$

where $\delta=\varepsilon / 2$. In particular, if the minimal degree $m(H)$ is unbounded, then $H$ is indistinguishable. 
Proof. Apply the upper bound of Proposition 1, written in the form

$$
D_{H} \leq \sum_{1 \neq h \in H}\left|h^{G}\right|^{-1 / 2}
$$

To evaluate this sum we use a result from [25], showing that, for $G=S_{n}$ and $h \in G$ of support $k$ we have $\left|h^{G}\right|>n^{a k}$ for any real $a<1 / 3$ and $n$ large enough (given $a$ ). Using this we obtain

$$
D_{H}<\sum_{k \geq m(H)}\left|H_{k}\right| n^{-b k},
$$

for any real number $b<1 / 6$ and sufficiently large $n$. Let $\delta=\varepsilon / 2, b=1 / 6-\delta$, and $m=m(H)$. Then the upper bound on $\left|H_{k}\right|$ yields

$$
D_{H}<\sum_{k \geq m} n^{(1 / 6-\varepsilon) k} n^{-(1 / 6-\delta) k}=\sum_{k \geq m} n^{-\delta k} \leq 2 n^{-\delta m} .
$$

This proves the first assertion. Assuming $m=m(H)$ is unbounded, we see that $D_{H}$ is smaller than any fixed negative power of $n$, and so $H$ is indistinguishable.

Lemma 7. Let $H<S_{n}$ be primitive and $H \neq A_{n}, S_{n}$. Then for sufficiently large $n$ and for all $k$ we have $\left|H_{k}\right| \leq n^{\frac{k}{7}}$.

Proof. We use Babai's lower bound on the minimal degree of primitive subgroups $H \neq A_{n}, S_{n}$ [1], showing that

$$
m(H) \geq(\sqrt{n}-1) / 2 .
$$

Furthermore, we apply a theorem of Cameron [4] (which in turn relies on the Classification of Finite Simple Groups) describing all primitive groups of 'large' order. In particular it follows from that description that, for all large $n$, and for a primitive subgroup $H \neq A_{n}, S_{n}$, either

(i) $|H| \leq n^{c n^{1 / 3}}$, or

(ii) $n=\left(\begin{array}{l}l \\ 2\end{array}\right)$ for some $l$, and $H \leq S_{l}$ acting on 2-subsets of $\{1, \ldots, l\}$, or

(iii) $n=l^{2}$ for some $l$, and $H \leq S_{l} 2 S_{2}$ acting on $\{1, \ldots, l\}^{2}$ in the so called product action.

We claim that for all large $n$ and for all $k$ we have $\left|H_{k}\right| \leq n^{k / 7}$. To show this it suffices to consider $k \geq(\sqrt{n}-1) / 2$, otherwise $\left|H_{k}\right|=0$ by (3). Now, if $H$ satisfies condition (i) above then the claim follows trivially using $\left|H_{k}\right| \leq|H|$. So it remains to consider groups $H$ in cases (ii) and (iii). Here a simple computation based on the known actions of $H$ completes the proof of the lemma.

Theorem 5 now follows by combining the above two lemmas. In fact we obtain, for all primitive subgroups $H \neq A_{n}, S_{n}$,

$$
D_{H} \leq 2 n^{-m(H) / 84} \leq 2 n^{-(\sqrt{n}-1) / 168} .
$$


The remainder of this section is devoted to reducing Theorem C to Theorem B.

Lemma 8. Let $C$ be a conjugacy class in $S_{n}$ consisting of elements of support $k$. Then $|C| \geq c\left(\begin{array}{l}n \\ k\end{array}\right) \sqrt{k !} \cdot k^{-\frac{1}{2}}$, where $c$ is an absolute positive constant.

Proof. There are $\left(\begin{array}{l}n \\ k\end{array}\right)$ ways to chose the subset $S \subset\{1, \ldots, n\}$ of letters moved by an element $h \in C$. Given the subset $S,\left.h\right|_{S}$ is a fixed point free permutation of degree $k$. The number of such permutations with a given cycle structure is minimal in the case of a fixed point free involution and is in this case equal to $k ! / 2^{\frac{k}{2}}(k / 2)$ !. Using Stirling's formula, we see that this expression is at least $c \sqrt{k !} \cdot k^{-\frac{1}{2}}$. Putting everything together the lemma follows.

Lemma 9. Let $H \leq S_{n}$. Then

$$
D_{H} \leq a \sum_{1 \leq k \leq n}\left|H_{k}\right|\left(\begin{array}{l}
n \\
k
\end{array}\right)^{-\frac{1}{2}}(k !)^{-\frac{1}{4}} \cdot k^{\frac{1}{4}},
$$

where a is some absolute constant.

Proof. We use part 2 of Proposition 1:

$$
D_{H} \leq \sum_{1 \neq h \in H}\left|h^{G}\right|^{-1 / 2} .
$$

By Lemma 8 we conclude that

$$
\sum_{h \in H_{k}}\left|h^{G}\right|^{-\frac{1}{2}} \leq c^{-1 / 2}\left|H_{k}\right|\left(\begin{array}{l}
n \\
k
\end{array}\right)^{-\frac{1}{2}}(k !)^{-\frac{1}{4}} \cdot k^{\frac{1}{4}} .
$$

The result follows.

Suppose now that Theorem B holds and let $m=m(H)$. Substituting

$$
\left|H_{k}\right| \leq n^{-\varepsilon m}\left(\begin{array}{l}
n \\
k
\end{array}\right)^{\frac{1}{2}}(k !)^{\frac{1}{4}}
$$

in Lemma 9 we obtain

$$
D_{H} \leq a n^{-\varepsilon m} \sum_{1 \leq k \leq n} k^{\frac{1}{4}} \leq a n^{-\varepsilon m} \cdot n^{\frac{5}{4}} .
$$

Therefore, if $m$ is unbounded, $D_{H}$ is smaller than any inverse polynomial in $n$, and hence $H$ is indistinguishable. Moreover, assuming $D_{H} \geq n^{-c}$ (and $n^{3 / 4} \geq a$ as we may) we obtain $\varepsilon m-2 \leq c$, and so

$$
m \leq 2 / \varepsilon+c / \varepsilon
$$

Hence, Theorem C follows from Theorem B. 


\section{Bounds on the group size in terms of the minimal degree}

In this section we prove Theorem A. It extends a theorem of Martin Liebeck [22], [23] which bounds the order of transitive groups with large minimal degree.

We call $H$ a subdirect product subgroup of $S^{t}$ if it is a subdirect product of $S_{1} \times \cdots \times S_{t}$ where all the $S_{i}$ are isomorphic to $S$. Such an $H$ is called a diagonal subgroup if it is isomorphic to $S$.

Lemma 10. Let $S$ be a non-Abelian simple group and $H$ a subdirect product subgroup of $S^{t} \cong S_{1} \times \cdots \times S_{t}$.

1) Then there is a partition of the set of indices $\{1, \ldots, t\}$ and for each part, say $\left\{i_{j_{1}}, \ldots, i_{j_{k}}\right\}$, a diagonal subgroup $D_{j}$ of $S_{i_{j_{1}}} \times \cdots \times S_{i_{j_{k}}}$ such that $H$ is a direct product of the subgroups $D_{j}$.

2) Assume that $S \cong \operatorname{Alt}(k)$ for some $k \geq 7$ and let $D$ be a diagonal subgroup of $S^{t}$. Let $d=\left(d_{1}, \ldots, d_{t}\right)$ be an element of $D$ such that $d_{1}$ is a 3-cycle. Then all the $d_{i}$ are 3 -cycles.

Proof. 1. This is a standard result.

2. This follows from the fact that the set of 3-cycles is invariant under automorphisms of $\operatorname{Alt}(k)$ if $k \geq 7$ [5, Lemma 8.2. A].

Let $H$ be a permutation group with minimal degree $m=m(H)$. Denote by $\Omega_{1}, \ldots, \Omega_{r}$ the orbits of $H$ and set $t=\max \left|\Omega_{i}\right|$. Let $\mathcal{B}_{i}=\left\{B_{i_{1}}, \ldots, B_{i_{k_{i}}}\right\}$ be a system of blocks of imprimitivity for the action of $H$ on $\Omega_{i}$ such that $k_{i}>1$ is minimal (if $H$ acts on $\Omega_{i}$ as a primitive group, then $k_{i}=\left|\Omega_{i}\right|$ ). Denote by $K_{i}$ the kernel of the action of $H$ on $\mathscr{B}_{i}$ and the size of the blocks in $\mathscr{B}_{i}$ by $b_{i}$. Set $\mathscr{B}=\bigcup_{i=1}^{r} \mathscr{B}_{i}, K=\bigcap_{i=1}^{r} K_{i}$ and $x=\sum_{i=1}^{r}\left(k_{i}-1\right)$. Note that $K$ has at least $r+x$ orbits.

Proposition 11. $|H / K| \leq 5^{x} t^{3 n / m}$.

Proof. $H$ acts on $\mathscr{B}_{i}$ as a primitive permutation group $P_{i} \cong H / K_{i}$ of degree $k_{i}$. If $P_{i}$ does not contain Alt $\left(k_{i}\right)$, then, by a result of Praeger and Saxl, [31] we have $\left|P_{i}\right| \leq 4^{k_{i}}$. Together with some trivial computation for small values of $k_{i}$ this implies $\left|P_{i}\right| \leq 5^{k_{i}-1}$.

Denote by $S$ the intersection of all the $K_{i}$ for which $\left|P_{i}\right| \leq 5^{k_{i}-1}$ holds. Then $S$ acts on each $\mathcal{B}_{i}$ either as a trivial group or as a group containing $\operatorname{Alt}\left(k_{i}\right)$ where $k_{i} \geq 7$. Without loss of generality one can assume that $S$ acts trivially on $\mathscr{B}_{i}$ exactly if $i>q$. The group $A=(S / K)^{\prime}$ is a subdirect product subgroup of $\operatorname{Alt}\left(k_{1}\right) \times \cdots \times \operatorname{Alt}\left(k_{q}\right)$. Denoting by $\bar{A}$ the inverse image of $A$ in $S$ we see that $|H / \bar{A}| \leq 5^{x}$ holds.

To complete the proof it is enough to show that

$$
|\bar{A} / K|=|A| \leq t^{3 n / m} .
$$


It follows from Lemma 10 that $A$ is a direct product of diagonal subgroups $A_{j}$. Each $A_{j}$ acts as an alternating group $\operatorname{Alt}\left(n_{j}\right)$ on some systems of blocks $\mathscr{B}_{i}$ with $n_{j}=k_{i}$, trivially on the rest and is isomorphic to $\operatorname{Alt}\left(n_{j}\right)$.

We claim that the sum of the block-sizes $b_{i}$ corresponding to $A_{j}$ is at least $m / 3$. To simplify notation we assume that $A_{j}$ acts trivially on $\mathscr{B}_{i}$ exactly if $i>p$. By Lemma 10 there is an element $a_{j}$ of $A_{j}$ which acts as a 3 -cycle on each $\mathscr{B}_{i}$ for $i \leq p$. This element corresponds to an element $\overline{a_{j}}$ of $\bar{A}$ which moves at most $3 \sum_{i=1}^{p} b_{i}$ elements. Hence $3 \sum_{i=1}^{p} b_{i} \geq m$ as claimed.

It follows that each $A_{j}$ moves at least $n_{j} m / 3$ points. This implies that the sum of the $n_{j}$ for all diagonal subgroups $A_{j}$ is at most $3 n / m$. Each $A_{j}$ has order $\frac{1}{2} n_{j} ! \leq t^{n_{j}}$. Hence $|A| \leq t^{3 n / m}$ as required.

We are now ready to prove Theorem A:

Proof of Theorem A. Set $\ell=\min \left(m, \log _{2} n\right)$. We have to show that $|G| \leq n^{10 \frac{n}{\ell}}$. Denote by $\Delta_{1}, \ldots, \Delta_{t}$ the orbits of $G$. Let $D_{i}=\left\{D_{i 1}, \ldots, D_{i h_{i}}\right\}$ be a system of blocks of imprimitivity for the action of $G$ on $\Delta_{i}$, such that the block size $d_{i}$ is at least $\ell$ and $d_{i}$ is as small as possible with this restriction (if there are no proper blocks of size $\geq \ell$ then we set $D_{i 1}=\Delta_{i}$ ). $G$ acts on $\mathscr{D}=\bigcup_{i=1}^{t} D_{i}$ as a permutation group of degree at most $n / \ell$. Hence the kernel $H$ of the action has index $\leq n^{\frac{n}{\ell}}$ in $G$.

Denote by $\Omega_{1}, \ldots, \Omega_{r}$ the orbits of $H$ and let $\mathscr{B}_{1}, \ldots, \mathscr{B}_{r}$ be systems of imprimitivity as in Proposition 11. By the construction of $H$ it is clear that we have $b_{i}<\ell$ for each $i$. Applying Proposition 11 we obtain a subgroup $K$ of index $\leq 5^{x} n^{3 n / m}$ such that $K$ has at least $r+x$ orbits and each orbit has size $<\ell$.

We apply Proposition 11 to $K$ to obtain a subgroup $K_{1}$ of index $\leq 5^{x_{1}} \cdot \ell^{3 n / m}$ in $K$, which has at least $r+x+x_{1}$ orbits, each of size $\leq \frac{\ell}{2}$.

Continuing in this fashion we obtain a descending series of subgroups $K>K_{1}>$ $K_{2}>\cdots>K_{v}=1$. The maximal size of an orbit of $K_{i}$ is at most $\ell / 2^{i}$, hence the above series of subgroups has length $v \leq \log _{2} \ell$.

Since $K_{i}$ has at least $r+x+x_{1}+\cdots+x_{i}$ orbits we have $x+x_{1}+\cdots+x_{v} \leq n$. Hence $|H|=|H / K| \cdot\left|K / K_{1}\right| \prod_{i=1}^{v-1}\left|K_{i} / K_{i+1}\right| \leq 5^{n} n^{3 n / m} \cdot\left(\ell^{3 n / m}\right)^{v} \leq 5^{n} \cdot n^{3 n / \ell}$. $2^{3 n\left(\frac{(\log \ell)^{2}}{\ell}\right)} \leq 5^{n} n^{3 n / \ell} \cdot 2^{3 n \cdot 9 / 8} \leq n^{3 n / \ell} \cdot 2^{6 n}$. Therefore we have $|G| \leq n^{4 n / \ell} \cdot 2^{6 n} \leq$ $n^{10 \frac{n}{\ell}}$ as required.

\section{Counting elements of given support}

This section, which is the longest in this paper, is devoted to the proof of Theorem B. The main ingredients of the proof are Theorem A and Proposition 5.

We will use the following inequality many times. 
Proposition 12. Let $x, y, n$ be positive integers such that $x+y \leq n$. Then $\left(\begin{array}{l}n \\ x\end{array}\right)\left(\begin{array}{l}n \\ y\end{array}\right) \leq$ $\left(\begin{array}{c}n \\ x+y\end{array}\right) 2^{2(x+y)}$ holds.

Proof. In fact we claim that the stronger inequality $\left(\begin{array}{l}n \\ x\end{array}\right)\left(\begin{array}{l}n \\ y\end{array}\right) \leq\left(\begin{array}{c}n \\ x+y\end{array}\right)\left(\begin{array}{c}x+y \\ y\end{array}\right)^{2}$ holds. This is equivalent to

$$
\frac{n(n-1) \ldots(n-x+1) n(n-1) \ldots(n-y+1)}{n(n-1) \ldots(n-x-y+1)} \leq\left(\begin{array}{c}
x+y \\
y
\end{array}\right)
$$

which is equivalent to

$$
\frac{n(n-1) \ldots(n-y+1)}{(n-x) \ldots(n-x-y+1)} \leq \frac{(x+y)(x+y-1) \ldots(x+1)}{y !}
$$

But this follows by multiplying the inequalities

$$
\frac{n-t}{n-x-t} \leq \frac{x+y-t}{y-t} \text { for } t=0,1, \ldots, y-1 .
$$

These latter inequalities follow from $x+y \leq n$.

To avoid some technical difficulties we first prove Theorem B directly in the case when $k$ is very large.

Lemma 13. Let $H$ be a permutation group of degree $n$ and minimal degree $m \geq$ 100000 . Assume that $k \geq n^{\frac{2}{3}+\frac{1}{100}}$ and $k \geq 2^{100000}$. Then there exists a constant $\varepsilon>0$ such that $\left|H_{k}\right| \leq\left(\begin{array}{l}n \\ k\end{array}\right)^{\frac{1}{2}}(k !)^{\frac{1}{4}} n^{-\varepsilon m}$ holds.

Proof. We have to count elements $h \in H$ with $\operatorname{supp}(h)=k$. There are at most $\left(\begin{array}{l}n \\ k\end{array}\right)$ choices for $\operatorname{supp}(h)$ and given this by Theorem A there are at most $k \frac{k}{10000}$ choices for $h$ itself. We have to show that

$$
\left(\begin{array}{l}
n \\
k
\end{array}\right) k^{\frac{k}{10000}} \leq\left(\begin{array}{l}
n \\
k
\end{array}\right)^{\frac{1}{2}}(k !)^{\frac{1}{4}} n^{-\varepsilon m} .
$$

This is equivalent to

$$
\left(\begin{array}{l}
n \\
k
\end{array}\right) k^{\frac{k}{5000} \cdot n^{2 \varepsilon m}} \leq(k !)^{\frac{1}{2}}
$$

which follows from

$$
n^{k} \cdot k^{\frac{k}{5000}} \cdot n^{2 \varepsilon k} \leq(k !)^{\frac{3}{2}} .
$$


This in turn is implied by

$$
n^{k} \cdot k^{\frac{k}{5000}} k^{3 \varepsilon k} \leq\left(\frac{k}{e}\right)^{\frac{3}{2} k}
$$

which reduces to

$$
n^{\frac{2}{3}}\left(e \cdot k^{\frac{1}{7500}+2 \varepsilon}\right) \leq k
$$

which follows from our conditions if $\varepsilon$ is small enough.

We now fix $a \geq 10000$ such that if $H$ is a primitive permutation group of degree $n \geq a$ not containing $\operatorname{Alt}(n)$, then $m(H) \geq 100$ and $\left|H_{k}\right| \leq n^{k / 7}$. This is possible by [1] and Lemma 7 above.

Next, we introduce some notation which will be used in the rest of this section. Let $G$ be a permutation group of degree $n$ with no fixed points. Denote by $\Omega_{1}, \Omega_{2}, \ldots$, the orbits of $G$. Let $\mathscr{B}_{i}=\left\{B_{i 1}, B_{i 2}, \ldots\right\}$ be a system of blocks of imprimitivity for the action of $G$ on $\Omega_{i}$, such that $\left|B_{i 1}\right| \geq 2$ is minimal. Then the setwise stabiliser of the blocks $B_{i j}$ in $G$ acts as some primitive group $P_{i j}$ on $B_{i j}$. The $P_{i j}$ are permutation equivalent for $i$ fixed.

We partition the set of blocks $\mathscr{B}=\bigcup \mathscr{B}_{i}$ into 3 subsets as follows. Denote by $\mathcal{S}=\left\{S_{1}, S_{2}, \ldots\right\}$ the set of blocks of size $<a$. Denote by $\mathcal{A}=\left\{A_{1}, A_{2}, \ldots\right\}$ the set of blocks $B_{i j}$ in $\mathscr{B} \backslash \mathcal{S}$ for which $P_{i j}$ contains $\operatorname{Alt}\left(B_{i j}\right)$, and denote by $\mathscr{L}=$ $\left\{L_{1}, L_{2}, \ldots,\right\}$ the set of the remaining blocks. Set $S=\bigcup S_{i}, A=\bigcup A_{i}$ and $L=\bigcup L_{i}$. It is clear that any $g \in G$ fixes the sets $S, L$ and $A$. We denote the action of $g \in G$ on a set $X$ (fixed by $g$ ) by $g_{X}$ and the action of $G$ on a fixed set $X$ by $G_{X}$.

Our next lemma shows that in a sense there are not too many possibilities for the action of some $g \in G$ on the set $S \cup L$.

Lemma 14. 1) The number of pairs $\left(\operatorname{supp}\left(g_{S}\right), g_{L}\right)$ for permutations $g$ with $|\operatorname{supp}(g)|=k,\left|\operatorname{supp}\left(g_{L}\right)\right|=x$ and $\left|\operatorname{supp}\left(g_{A}\right)\right|=y$ is at most $\left(\left[\frac{k-x-y}{2}\right]\right) 2^{a k}$.

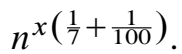

2) Given $\operatorname{supp}\left(g_{S}\right)$, the number of possible actions $g_{S}$ is at most $a^{k-x-y}\left[\frac{k-x-y}{2}\right]$ !. In fact this is an upper bound for the number of possible actions on $\operatorname{supp}\left(g_{S}\right)$ of elements $h$ which fix $\operatorname{supp}\left(g_{S}\right)$.

Proof. If $g$ moves a point of some block, then it moves at least two points of the block. Hence the number $t$ of blocks in $\delta$ which contain points from $\operatorname{supp}(g)$ is at most $\left[\frac{k-x-y}{2}\right]$. These blocks can be chosen in at most $\left(\begin{array}{l}n \\ t\end{array}\right)$ ways. Given these blocks the number of choices for $\operatorname{supp}\left(g_{S}\right)$ is at most $\left(2^{a}\right)^{t}$.

Note that $g_{S}$ (or $h \in G$ fixing $\left.\operatorname{supp}\left(g_{S}\right)\right)$ moves $a_{1}, a_{2}, \ldots, a_{t}$ given points of the chosen blocks in at most $a_{1} ! a_{2} ! \ldots a_{t} ! \cdot t ! \leq \prod_{i=1}^{t} a^{a_{i}} \cdot t ! \leq a^{k-x-y}\left[\frac{k-x-y}{2}\right]$ ! ways, proving 2). 
Each block in $\mathscr{L}$ which contains points of $\operatorname{supp}(g)$ contains at least 100 such points (by the choice of $a$, see the notation introduced after Lemma 13), hence the number $\ell$ of such blocks is at most $x / 100$. These blocks can be chosen in at most $\left(\begin{array}{l}n \\ \ell\end{array}\right) \leq n \frac{x}{100} / \ell !$ ways.

There are $\ell_{1} \leq \ell$ blocks from $\mathscr{L}$ fully contained in $\operatorname{supp}(g)$ and these can be chosen in at most $2^{\ell}$ ways.

By our assumption on the blocks in $\mathscr{L}$ and the Praeger-Saxl theorem [31] the stabilisers of a block $B_{i j}$ in $\mathscr{L}$ can act on the block in at most $4^{\left|B_{i j}\right|}$ ways. This implies that the stabiliser of the union of the above blocks can act on this union in at most $4^{x} \ell_{1}$ ! ways. Hence this is an upper bound for the number of actions of $g$ on the blocks contained in $\operatorname{supp}(g)$.

Assume that on the remaining blocks (which are as sets fixed by $g$ ) $g$ acts as a permutation of degree $x_{1}, x_{2}, \ldots$ The number $x_{1}, x_{2}, \ldots$ can be chosen in at most $2^{x}$ ways. Given these numbers the number of actions of $g$ on these remaining blocks can be chosen in at most $n^{x_{1} / 7} \cdot n^{x_{2} / 7} \cdots \leq n^{x / 7}$ ways by Lemma 7 .

Altogether the number of choices for $\operatorname{supp}\left(g_{S}\right)$ and $g_{L}$ is at most

$$
\left(\begin{array}{l}
n \\
t
\end{array}\right) 2^{a t}\left(n^{\frac{x}{100}} / \ell !\right) 2^{\ell} 4^{x} \ell_{1} ! 2^{x} \cdot n^{x / 7} \leq\left(\begin{array}{c}
n \\
{\left[\frac{k-x-y}{2}\right]}
\end{array}\right) n^{\frac{x}{7}+\frac{x}{100}} 2^{a k}
$$

as required.

Corollary 15. The number of pairs $\left(\operatorname{supp}\left(g_{S}\right), g_{L}\right)$ for permutations $g$ with $|\operatorname{supp}(g)|=k$ and $\left|\operatorname{supp}\left(g_{A}\right)\right|=y$ is at most

$$
\left(\begin{array}{l}
n \\
k
\end{array}\right)^{\frac{1}{2}}\left[\frac{y}{2}\right] ! n^{-\frac{y}{2}} \cdot 2^{(a+4) k} \quad \text { if } k \leq n^{\frac{2}{3}+\frac{1}{100}}
$$

and $n$ is sufficiently large.

Proof. We first claim that the number of permutations $g$ considered is at most $\frac{1}{n}\left(\begin{array}{c}n \\ {\left[\frac{k-y}{2}\right]}\end{array}\right) 2^{(a+1) k}$. By Lemma 14 it is sufficient to prove that for all $x \leq k$ we have

$$
\left(\begin{array}{c}
n \\
{\left[\frac{k-x-y}{2}\right]}
\end{array}\right) 2^{a k} n^{x\left(\frac{1}{7}+\frac{1}{100}\right)} \leq \frac{1}{k n}\left(\begin{array}{c}
n \\
{\left[\frac{k-y}{2}\right]}
\end{array}\right) 2^{(a+1) k} .
$$

This is obvious if $x=0$, otherwise we have $x \geq 100$. By Proposition 12

$$
\left(\begin{array}{c}
n \\
{\left[\frac{k-x-y}{2}\right]}
\end{array}\right)\left(\begin{array}{c}
n \\
{\left[\frac{x}{2}\right]}
\end{array}\right) \leq\left(\begin{array}{c}
n \\
{\left[\frac{k-y}{2}\right]}
\end{array}\right) 2^{k}
$$

holds, hence it is enough to show that $n^{x\left(\frac{1}{7}+\frac{1}{100}\right)+2} \leq\left(\begin{array}{c}n \\ {\left[\frac{x}{2}\right]}\end{array}\right)$. But this follows using $100 \leq x \leq k \leq n^{\frac{2}{3}+\frac{1}{100}}$. 
Using Proposition 12 we obtain that

$$
\begin{aligned}
\frac{1}{n}\left(\begin{array}{c}
n \\
{\left[\frac{k-y}{2}\right]}
\end{array}\right) 2^{(a+1) k} & \leq \frac{1}{n}\left(\begin{array}{c}
n \\
{\left[\frac{k}{2}\right]}
\end{array}\right)\left(\begin{array}{c}
n \\
{\left[\frac{y}{2}\right]}
\end{array}\right)^{-1} 2^{(a+2) k} \\
& \leq \frac{1}{n}\left(\begin{array}{l}
n \\
k
\end{array}\right)^{\frac{1}{2}}\left(\begin{array}{c}
n \\
{\left[\frac{y}{2}\right]}
\end{array}\right)^{-1} 2^{(a+3) k} \leq \frac{1}{n}\left(\begin{array}{l}
n \\
k
\end{array}\right)^{\frac{1}{2}}\left[\frac{y}{2}\right] ! n^{-\left[\frac{y}{2}\right]} 2^{(a+4) k} \\
& \leq\left(\begin{array}{l}
n \\
k
\end{array}\right)^{\frac{1}{2}}\left[\frac{y}{2}\right] ! n^{-\frac{y}{2}} 2^{(a+4) k}
\end{aligned}
$$

proving the corollary.

The most difficult part of the proof of Theorem B is when $y$ is large compared to $m$. The following result implies Theorem B in the case when this holds and moreover $k$ ! is large compared to $n^{y}$.

Lemma 16. Assume that $m \geq 100000, k \leq n^{\frac{2}{3}+\frac{1}{100}}, n^{3 y} \leq k$ ! and $k$ is suffciently large (in particular $k \geq 2^{100000}$ ). Then the number of permutations $g$ with $|\operatorname{supp}(g)|=k$ and $\left|\operatorname{supp}\left(g_{A}\right)\right|=y$ is at most $\left(\begin{array}{l}n \\ k\end{array}\right)^{\frac{1}{2}}(k !)^{\frac{1}{4}} n^{-\frac{y}{60}}$.

Proof. The number of choices for $\operatorname{supp}\left(g_{A}\right)$ is at most $\left(\begin{array}{l}n \\ y\end{array}\right)$. Hence by Corollary 15 the number of choices for $\operatorname{supp}(g)$ is at most

$$
\left(\begin{array}{l}
n \\
k
\end{array}\right)^{\frac{1}{2}}\left[\frac{y}{2}\right] ! n^{-\frac{y}{2}} 2^{(a+4) k}\left(\begin{array}{l}
n \\
y
\end{array}\right) \leq\left(\begin{array}{l}
n \\
k
\end{array}\right)^{\frac{1}{2}} n^{\frac{y}{2}} 2^{(a+4) k} .
$$

Using Theorem A we see that the number of choices for $g$ is at most

$$
\left(\begin{array}{l}
n \\
k
\end{array}\right)^{\frac{1}{2}} n^{\frac{y}{2}} 2^{(a+4) k} k^{\frac{k}{10000}} \leq\left(\begin{array}{l}
n \\
k
\end{array}\right)^{\frac{1}{2}}(k !)^{\frac{1}{5}} \cdot k^{\frac{k}{10000}} \cdot 2^{(a+4) k} \cdot n^{-\frac{y}{60}} .
$$

If $k$ is large enough (compared to the constant $a$ ) then $(k !) \frac{1}{20} \geq k^{\frac{k}{10000}} \cdot 2^{(a+4) k}$ and our statement holds.

Next we describe an important subgroup of $G$. Consider the set consisting of the points in $S$ and $L$ and the blocks in $\mathcal{A}$. Let $K$ be the kernel of the action of $G$ on this set. By definition $K$ fixes all the points outside $A$. Moreover, if $A_{i} \in \mathcal{A}$, then the action $K_{i}$ of $K$ on $A_{i}$ is a normal subgroup of the action of the stabiliser of $A_{i}$ in $G$, hence it is either $\operatorname{Sym}\left(A_{i}\right), \operatorname{Alt}\left(A_{i}\right)$ or 1 . 
Without loss of generality one can assume that $K$ acts trivially on $A_{i}$ exactly if $i>q$. Now $K$ is a subdirect product of the $K_{i}$, therefore its commutator subgroup $K^{\prime}$ is a subdirect product subgroup of Alt $\left(A_{1}\right) \times \cdots \times \operatorname{Alt}\left(A_{q}\right)$. Hence by Lemma $10 K^{\prime}$ is a direct product of diagonal subgroups $D_{j}$. Each $D_{j}$ acts as an alternating group Alt $\left(n_{j}\right)$ on some blocks $A_{i}$ of size $n_{j}$. By Lemma $10 D_{j}$ contains an element $d_{j}$ which acts as a 3-cycle on each of the corresponding $A_{i}$. Hence $D_{j}$ acts non-trivially on at least $\frac{m}{3}$ blocks $A_{i}$ (since $\left|\operatorname{supp}\left(d_{j}\right)\right| \geq m$ ). Now $K$ is a subgroup of the normaliser $N$ of $K^{\prime}$ in $\prod_{i=1}^{q} \operatorname{Sym}\left(A_{i}\right)$. Clearly $N$ is a direct product of groups $N_{j} \geq D_{j}$ where $N_{j}$ is isomorphic to $\operatorname{Sym}\left(n_{j}\right)$ and contains $D_{j} \cong \operatorname{Alt}\left(n_{j}\right)$ in a natural way.

Proposition 17. There are at most $n^{\frac{3 h}{m}}$ elements $g$ of $K$ with $|\operatorname{supp}(g)|=h$ (where $m=m(G))$.

Proof. We have a unique decomposition $g=g_{1} g_{2} \ldots$ where $g_{j} \in N_{j}$. Let us choose for each $j$ a block on which $N_{j}$ acts non-trivially. It is clear that $g_{j}$ is determined uniquely by its action on the chosen block. Therefore $g$ is determined by its action on the union $U$ of the chosen blocks.

It follows by the above discussion that $|\operatorname{supp}(g) \cap U| \leq \frac{3 h}{m}$. Hence the number of choices for $g$ is at most $|U|^{\frac{3 h}{m}} \leq n^{\frac{3 h}{m}}$.

Proposition 18. Assume that $m \geq 100000$. Then the number of permutations $g$ with $g_{S \cup L}$ fixed and $\operatorname{supp}\left(g_{A}\right)=y$ is at most $n^{y / 5000}$.

Proof. The coset $g K$ is determined by $g_{S \cup L}$ and the action of $g$ on the blocks in $\mathcal{A}$. Now $g$ can move at most $t \leq \frac{y}{a}$ blocks in $\mathcal{A}$.

The number of choices for these blocks is less than $\left(\begin{array}{c}n / a \\ t\end{array}\right)$ and given these blocks the number of ways $g$ can act on them is at most $t$ !. Hence $g$ can act in at most $\left(\frac{n}{a}\right)^{\left[\frac{y}{a}\right]}+\left(\frac{n}{a}\right)^{\left[\frac{y}{a}\right]-1}+\cdots \leq n^{\frac{y}{a}}$ ways on $\mathcal{A}$. If $g K$ contains another element $f$ with $|\operatorname{supp}(f)| \stackrel{=}{=}$ and $\left|\operatorname{supp}\left(f_{A}\right)\right|=y$, then $g f^{-1} \in K$ and $\mid\left(\operatorname{supp}\left(g f^{-1}\right) \mid \leq 2 y\right.$. Hence by Proposition 17 there are at most $n^{\frac{6 y}{m}} \leq n \frac{y}{10000}$ such elements $g f^{-1}$. Of course $g$ and $g f^{-1}$ determines $f$. Altogether we see that the number of elements $g$ considered is at most $n \frac{y}{5000}$.

Remark. As the proof shows (see also the proof of Proposition 17 and the preceding discussion) the conclusion of Proposition 18 holds under the much weaker assumption that all elements of order 3 in $G$ move at least 100000 points.

Proposition 19. Assume that $m \geq 100000, k \leq n^{\frac{2}{3}+\frac{1}{100}}, y \neq 0$ and $n$ is sufficiently large. Then the number of permutations $g \in G$ with $|\operatorname{supp}(g)|=k$ and $\left|\operatorname{supp}\left(g_{A}\right)\right|=y$ is at most $\left(\begin{array}{l}n \\ k\end{array}\right)^{\frac{1}{2}} n^{-\frac{y}{2}+\frac{y}{5000}} k ! 2^{(a+4) k}$. 
Proof. By Corollary 15 the number of possibilities for $\operatorname{supp}\left(g_{S \cup L}\right)$ is at most $\left(\begin{array}{l}n \\ k\end{array}\right)^{\frac{1}{2}}\left[\frac{y}{2}\right] ! n^{-\frac{y}{2}} \cdot 2^{(a+4) k}$. Therefore the number of possibilities for $g_{S \cup L}$ is at most

$$
\left(\begin{array}{l}
n \\
k
\end{array}\right)^{\frac{1}{2}} n^{-\frac{y}{2}} 2^{(a+4) k}\left[\frac{y}{2}\right] !(k-y) ! \leq\left(\begin{array}{l}
n \\
k
\end{array}\right)^{\frac{1}{2}} n^{-\frac{y}{2}} 2^{(a+4) k} \cdot k ! .
$$

Hence by Proposition 18 the number of choices for $g$ is at most

$$
\left(\begin{array}{l}
n \\
k
\end{array}\right)^{\frac{1}{2}} n^{-\frac{y}{2}+\frac{y}{5000} \cdot k ! 2^{(a+4) k}}
$$

as required.

The next result as a counterpart of Lemma 16 deals with the case when $n^{y}$ is large compared to $k$ ! (and $y$ is large compared to $m$ ).

Corollary 20. Assume that $k \leq n^{\frac{2}{3}+\frac{1}{100}}, n^{\frac{y}{8}} \geq k$ ! and $m$ is sufficiently large. Then the number of permutations $g$ with $|\operatorname{supp}(g)|=k$ and $\operatorname{supp}\left(g_{A}\right) \mid=y$ is at most $\left(\begin{array}{l}n \\ k\end{array}\right)^{\frac{1}{2}} n^{-\frac{y}{5}}$.

Proof. We have $m \leq k \leq n$, hence if $m$ is large enough Proposition 19 is applicable. Moreover, we have $2^{(a+4) k} \leq k$ ! if $m$ is large enough (compared to the fixed constant $a$ ).

Hence in this case we have

$$
\left(\begin{array}{l}
n \\
k
\end{array}\right)^{\frac{1}{2}} n^{-\frac{y}{2}+\frac{y}{5000}}\left(k ! 2^{(a+4) k}\right) \leq\left(\begin{array}{l}
n \\
k
\end{array}\right)^{\frac{1}{2}} n^{-\frac{y}{2}+\frac{y}{5000}+\frac{y}{4}} \leq\left(\begin{array}{l}
n \\
k
\end{array}\right)^{\frac{1}{2}} n^{-\frac{y}{5}} .
$$

To deal with the case when $k$ ! and $n^{y}$ are "almost equal" we have to introduce further ideas and notation. We call a pair of the form $\left(\operatorname{supp}\left(g_{S}\right), g_{L}\right)$ thick if the elements $g$ which correspond to it act in at least $(k !)^{\frac{1}{6}}$ different ways on $\operatorname{supp}\left(g_{S}\right)$ and call a pair thin otherwise.

Proposition 21. Assume that $m \geq 100000,2^{200 a} \leq k \leq n^{\frac{2}{3}+\frac{1}{100}}, y \neq 0$ and $n$ is sufficiently large. Then the number of permutations $g$ with $|\operatorname{supp}(g)|=k$ and $\left|\operatorname{supp}\left(g_{A}\right)\right|=y$ for which $\left(\operatorname{supp}\left(g_{S}\right), g_{L}\right)$ is thin is at most $\left(\begin{array}{l}n \\ k\end{array}\right)^{\frac{1}{2}}(k !)^{\frac{1}{6}+\frac{1}{100}}$.

Proof. By Corollary 15 the number of possibilities for the pair $\left(\operatorname{supp}\left(g_{S}\right), g_{L}\right)$ is at most $\left(\begin{array}{l}n \\ k\end{array}\right)^{\frac{1}{2}}\left[\frac{y}{2}\right] ! n^{-\frac{y}{2}} \cdot 2^{(a+4) k}$. Hence the number of possibilities for $g_{S \cup L}$ is at most

$$
\left(\begin{array}{l}
n \\
k
\end{array}\right)^{\frac{1}{2}}\left[\frac{y}{2}\right] ! n^{-\frac{y}{2}} \cdot 2^{(a+4) k}(k !)^{\frac{1}{6}} \leq\left(\begin{array}{l}
n \\
k
\end{array}\right)^{\frac{1}{2}}\left[\frac{y}{2}\right] ! n^{-\frac{y}{2}}(k !)^{\frac{1}{6}+\frac{1}{100}}
$$


(we used the condition $200 a \leq \log k$ ). Using Proposition 18 we see that the total number of elements $g$ considered is at most

$$
\left(\begin{array}{l}
n \\
k
\end{array}\right)^{\frac{1}{2}}(k !)^{\frac{1}{6}+\frac{1}{100}} n^{-\frac{y}{2}+\frac{y}{5000}}\left[\frac{y}{2}\right] ! \leq\left(\begin{array}{l}
n \\
k
\end{array}\right)^{\frac{1}{2}}(k !)^{\frac{1}{6}+\frac{1}{100}}
$$

(using $y \leq k \leq n^{\frac{2}{3}+\frac{1}{100}}$ ).

Proposition 22. Let $\left(\operatorname{supp}\left(g_{S}\right), g_{L}\right)$ be a thick pair. Denote the action of (the stabiliser of $\operatorname{supp}\left(g_{S}\right)$ in) $G$ on $\operatorname{supp}\left(g_{S}\right)$ by $H$. There is an element $\gamma$ which corresponds to this pair such that the centraliser of $\gamma_{S}$ in $H$ has order at most

$$
(5 a)^{k-x-y}\left[\frac{k-x-y}{2}\right] ! /(k !)^{\frac{1}{6}} .
$$

Proof. By Lemma 14 (2) $H$ has order at most $a^{k-x-y}\left[\frac{k-x-y}{2}\right]$ !. By a result of Kovács and Robinson [19] the number $k(H)$ of conjugacy classes of the permutation group $H$ is at most $5^{k-x-y}$. Using a well-known identity we obtain

$$
\sum_{h \in H} C_{H}(h)=k(H)|H| \leq(5 a)^{k-x-y}\left[\frac{k-x-y}{2}\right] .
$$

Since by definition we have at least $(k !)^{\frac{1}{6}}$ choices for $g_{S} \in H$, at least one of them has small centraliser as required.

Proposition 23. Assume that $m \geq 100000, k \geq 2^{100000}$ and $y \neq 0$. Let $\left(\operatorname{supp}\left(g_{S}\right), g_{L}\right)$ be a thick pair and $\gamma$ a corresponding permutation with small centraliser as above. The number of elements $g$ which correspond to this pair and satisfy the condition

$$
\left|\operatorname{supp}\left(g_{A}\right) \cap \operatorname{supp}\left(\gamma_{A}\right)\right| \geq \frac{y}{100}
$$

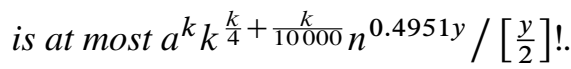

Proof. The number of choices for the set $\operatorname{supp}\left(g_{A}\right) \cap \operatorname{supp}\left(\gamma_{A}\right)$ is less than $2^{y}$. The number of choices for the rest of $\operatorname{supp}\left(g_{A}\right)$ is at most $\left(\begin{array}{c}n \\ {\left[\begin{array}{ll}0.99 & y\end{array}\right]}\end{array}\right)$. Given these sets (and hence $\operatorname{supp}(g))$ by Theorem A the number of choices for $g$ is at most $k \frac{k}{10000}$. It follows that the number of choices for $g$ is less than

$$
2^{k} n^{0.99 y} k^{\frac{k}{10000}} /\left[\frac{y}{2}\right] !
$$

Another estimate for the number of possible choices for $g$ is the following. The number of choices for $g_{S \cup L}$ is at most $a^{k-x-y}\left[\frac{k-x-y}{2}\right]$ ! by Lemma 14 (2). Hence 
by Proposition 18 the number of choices for $g$ is less than

$$
a^{k-x-y}\left[\frac{k-x-y}{2}\right] ! n \frac{y}{5000} \leq a^{k} k^{\frac{k}{2}} n^{\frac{y}{5000}} /\left[\frac{y}{2}\right] ! .
$$

A third estimate follows immediately from these; the number of choices for $g$ is at most

$$
\left(a^{k} \cdot k^{\frac{k}{2}} n^{\frac{y}{5000}} \cdot 2^{k} \cdot n^{0.99 y} \cdot k^{\frac{k}{10000}}\right)^{\frac{1}{2}} /\left[\frac{y}{2}\right] ! \leq a^{k} k^{\frac{k}{4}+\frac{k}{10000}} n^{0.4951 y} /\left[\frac{y}{2}\right] !
$$

as required.

Proposition 24. Assume that $m \geq 100000$ and $k \geq 2^{100000}$. Let $\left(\operatorname{supp}\left(g_{S}\right), g_{L}\right)$ be a thick pair and $\gamma$ a corresponding permutation with small centralizer (as in Proposition 22). The number of elements $g$ which correspond to this pair and satisfy

$$
\left|\operatorname{supp}\left(g_{A}\right) \cap \operatorname{supp}\left(\gamma_{A}\right)\right| \leq \frac{y}{100}
$$

is at most

$$
n^{\frac{y}{30}} \cdot k^{\frac{k}{3}+\frac{k}{10000}}(5 a)^{k} /\left[\frac{y}{2}\right] ! .
$$

Proof. Let us consider the commutator $[\gamma, g]$. By [5, Exercise 1.6.7] we have

$$
|\operatorname{supp}([\gamma, g]) \cap A| \leq 3\left|\operatorname{supp}\left(g_{A}\right) \cap \operatorname{supp}\left(\gamma_{A}\right)\right| \leq \frac{3 y}{100} .
$$

Hence the number of choices for $\operatorname{supp}([\gamma, g]) \cap A$ is at most $n^{\frac{3 y}{100}}$. Note that $\operatorname{supp}([\gamma, g]) \cap(S \cup L) \leq \operatorname{supp}\left(\gamma_{S \cup L}\right)$ (which is fixed). Using Theorem A we obtain that the number of choices for $[\gamma, g]$ is at most $n \frac{3 y}{100} \cdot k \frac{k}{10000}$. This commutator, together with $\gamma$, determines $g^{-1} \gamma g=\gamma[\gamma, g]$. If $h$ is another element with $h^{-1} \gamma h=g^{-1} \gamma g$, then $g h^{-1}$ centralises $\gamma$. Hence by the choice of $\gamma$ in Proposition 22) the number of possibilities for $h_{S}$ is less than

$$
(5 a)^{k}\left[\frac{k-y}{2}\right] ! /(k !)^{\frac{1}{6}} .
$$

Hence we have at most $n \frac{3 y}{100} \cdot k \frac{k}{10000}(5 a)^{k}\left[\frac{k-y}{2}\right] ! /(k !)^{\frac{1}{6}}$ choices for $g_{S \cup L}$ and given this, the number of choices for $g$ is at most $n \frac{y}{5000}$ by Proposition 18. Therefore the number of choices for $g$ is at most

$$
n^{\frac{3 y}{100}} \cdot n^{\frac{y}{5000}}(5 a)^{k} k^{\frac{k}{10000}}\left[\frac{k}{2}\right] ! /(k !)^{\frac{1}{6}}\left[\frac{y}{2}\right] ! \leq n^{\frac{y}{30}} \cdot k^{\frac{k}{3}+\frac{k}{10000}}(5 a)^{k} /\left[\frac{y}{2}\right] !
$$

Our next result which builds on most of the earlier ones in this section implies Theorem B if $y$ is large compared to $m$. 


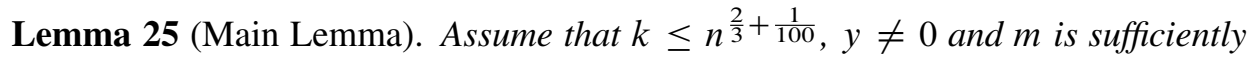
large. Then the number of permutations $g$ with $|\operatorname{supp}(g)|=k$ and $\left|\operatorname{supp}\left(g_{A}\right)\right|=y$ is at most $\left(\begin{array}{l}n \\ k\end{array}\right)^{\frac{1}{2}}(k !)^{\frac{1}{4}} n^{-\frac{y}{200}}$.

Proof. By Lemma 16 and Corollary 20 we may assume that $n^{3 y} \geq k ! \geq n^{\frac{y}{8}}$. By Proposition 21 the number of permutations $g$ with a thin pair $\left(\operatorname{supp}\left(g_{S}\right), g_{L}\right)$ is at most

$$
\left(\begin{array}{l}
n \\
k
\end{array}\right)^{\frac{1}{2}}(k !)^{\frac{1}{6}+\frac{1}{100}} \leq\left(\begin{array}{l}
n \\
k
\end{array}\right)^{\frac{1}{2}}(k !)^{\frac{1}{4}}(k !)^{-\frac{1}{20}} \leq\left(\begin{array}{l}
n \\
k
\end{array}\right)^{\frac{1}{2}}(k !)^{\frac{1}{4}} n^{-\frac{y}{160}} .
$$

It remains to bound the number of permutations $g$ with a thick pair. By Corollary 15 the number of possibilities for $\left(\operatorname{supp}\left(g_{S}\right), g_{L}\right)$ is at most $\left(\begin{array}{l}n \\ k\end{array}\right)^{\frac{1}{2}}\left[\frac{y}{2}\right] ! n^{-\frac{y}{2}} \cdot 2^{(a+4) k}$. Given this, by Propositions 23 and 24 the number of choices for $g$ is at most

$$
\begin{aligned}
& \left(a^{k} k^{\frac{k}{4}+\frac{k}{10000}} n^{0.4951 y}+(5 a)^{k} k^{\frac{k}{3}+\frac{k}{10000}} n^{\frac{y}{30}}\right) /\left[\frac{y}{2}\right] ! \\
& \leq(10 a)^{k}(k !)^{\frac{1}{4}} n^{\frac{3 y}{10000}}\left(n^{0.4951 y}+n^{\frac{y}{4}} \cdot n^{\frac{y}{30}}\right) /\left[\frac{y}{2}\right] ! \\
& \leq(10 a)^{k}(k !)^{\frac{1}{4}} n^{0.4954 y} /\left[\frac{y}{2}\right] \text { ! }
\end{aligned}
$$

(we used the inequality $n^{3 y} \geq\left(\frac{k}{e}\right)^{k}$ ). Hence the total number of permutations $g$ with a thick pair is at most $\left(\begin{array}{l}n \\ k\end{array}\right)^{\frac{1}{2}}(k !)^{\frac{1}{4}} n^{-0.006 y}\left((10 a)^{k} 2^{(a+4) k}\right)$. If $m$ and hence $k$ is large enough, then

$$
(10 a)^{k} 2^{(a+4) k} \leq \frac{1}{2}(k !)^{\frac{1}{3000}} \leq \frac{1}{2} n^{\frac{y}{1000}} .
$$

Our statement follows.

Next we prove Theorem B in the case when $x$ is large compared to $m$.

Proposition 26. Assume that $x \neq 0, n^{\frac{2}{3}+\frac{1}{100}} \geq k \geq 2^{100000}$ and $m$ is sufficiently large. Then the number of permutations $g$ with $|\operatorname{supp}(g)|=k,\left|\operatorname{supp}\left(g_{L}\right)\right|=x$ and $\left|\operatorname{supp}\left(g_{A}\right)\right|=y$ is at most $\left(\begin{array}{l}n \\ k\end{array}\right)^{\frac{1}{2}}(k !)^{\frac{1}{4}} n^{-\frac{x}{20000}}$.

Proof. If $y \geq \frac{x}{100}$, then our statement follows from the Main Lemma. Assume now that $y \leq \frac{x}{100}$. By Lemma 14 the number of choices for $\operatorname{supp}(g)$ is at most $\left(\left[\frac{k-x-y}{2}\right]\right) 2^{a k} n^{x\left(\frac{1}{7}+\frac{1}{100}\right)} \cdot\left(\begin{array}{l}n \\ y\end{array}\right)$. Hence, by Theorem A the number of choices for $g$ is at

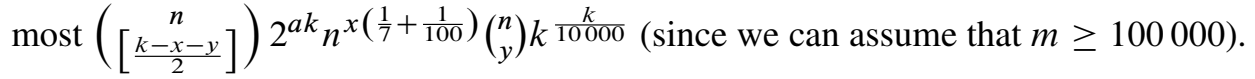


Using Proposition 12 and $y \leq \frac{x}{100}$ we see that this is at most

$$
\begin{gathered}
\left(\begin{array}{l}
n \\
k
\end{array}\right)^{\frac{1}{2}} 2^{(a+2) k} k^{\frac{k}{10000}} n^{x\left(\frac{1}{7}+\frac{1}{100}\right)}\left(\begin{array}{l}
n \\
y
\end{array}\right) /\left(\begin{array}{c}
n \\
{\left[\frac{x+y}{2}\right]}
\end{array}\right) \\
\leq\left(\begin{array}{l}
n \\
k
\end{array}\right)^{\frac{1}{2}} 2^{(a+2) k} k^{\frac{k}{10000}} n^{x\left(\frac{1}{7}+\frac{2}{100}\right)} /\left(\begin{array}{c}
n \\
{\left[\frac{x}{2}\right]}
\end{array}\right) .
\end{gathered}
$$

If $m$ and hence $k$ is large enough compared to $a$, we have $2^{(a+2) k} k \frac{k}{10000} \leq(k !)^{\frac{1}{4}}$. Using $100 \leq x \leq k \leq n^{\frac{2}{3}+\frac{1}{100}}$ we see that $n^{x\left(\frac{1}{7}+\frac{2}{100}\right)} /\left(\begin{array}{c}n \\ {\left[\frac{x}{2}\right]}\end{array}\right) \leq n^{-\frac{x}{100}}$. Our statement follows.

Let us return to the notation introduced after Lemma 13. If $S_{i} \in \delta$ is a small block, such that $g$ moves at least 3 points of $S_{i}$, then we denote $\left|\operatorname{supp}(g) \cap S_{i}\right|$ by $z_{i}$. We set $z(g)=\sum z_{i}$ (for all such $i$ ).

Proposition 27. Assume that $z \neq 0, n^{\frac{2}{3}+\frac{1}{100}} \geq k \geq 2^{100000}$ and $m$ is sufficiently large. Then the number of permutations $g$ with $z(g)=z$ is at most $\left(\begin{array}{l}n \\ k\end{array}\right)^{\frac{1}{2}}(k !)^{\frac{1}{4}} n^{-\frac{z}{800000}}$.

Proof. If $x \geq \frac{z}{40}$ or $y \geq \frac{z}{80}$, then our statement follows from Lemma 25 and Proposition 26. Assume otherwise. If $g$ moves a point of some block, then it moves at least two points of the block. Hence the number of blocks in $\delta$ which contain two points from $\operatorname{supp}(g)$ is at most $\left[\frac{k-z}{2}\right]$. These blocks can be chosen in at most $\left(\left[\frac{k-z}{2}\right]\right)$ ways. The blocks in $\delta$ which contain at least 3 points from $\operatorname{supp}(g)$ can be chosen in at most $\left(\begin{array}{c}n \\ {\left[\begin{array}{c}3 \\ 3\end{array}\right]}\end{array}\right)$ ways. Given these blocks the number of choices for $\operatorname{supp}\left(g_{S}\right)$ is at most

$$
\left(\begin{array}{c}
n \\
{\left[\frac{k-z}{2}\right]}
\end{array}\right)\left(\begin{array}{c}
n \\
{\left[\frac{z}{3}\right]}
\end{array}\right) 2^{a z} a^{2\left[\frac{k-z}{2}\right]} \leq\left(\begin{array}{c}
n \\
k
\end{array}\right)^{\frac{1}{2}} 2^{2 k} \cdot 2^{a k}\left(\begin{array}{c}
n \\
{\left[\frac{z}{3}\right]}
\end{array}\right) /\left(\begin{array}{c}
n \\
\left\lceil\frac{z}{2}\right\rceil
\end{array}\right) .
$$

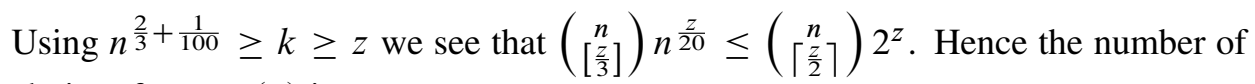
choices for $\operatorname{supp}(g)$ is at most

$$
\left(\begin{array}{l}
n \\
k
\end{array}\right)^{\frac{1}{2}} 2^{(a+3) k} n^{-\frac{z}{20}} n^{x+y} \leq\left(\begin{array}{l}
n \\
k
\end{array}\right)^{\frac{1}{2}} 2^{(a+3) k} n^{-\frac{z}{80}} .
$$

The number of choices for $g$ itself is at most $\left(\begin{array}{l}n \\ k\end{array}\right)^{\frac{1}{2}} n^{-\frac{z}{80}} 2^{(a+3) k} k \frac{k}{10000}$ which is less than $\left(\begin{array}{l}n \\ k\end{array}\right)^{\frac{1}{2}} n^{-\frac{z}{80}}(k !)^{\frac{1}{4}}$ if $k$ is large enough. 
Denote the number of small blocks $S_{i} \in \delta$ fixed by $g$ such that $\left|\operatorname{supp}(g) \cap S_{i}\right|=2$ by $v(g)$. On these blocks $g$ acts as a transposition.

Proposition 28. Assume that $n^{\frac{2}{3}+\frac{1}{100}} \geq k \geq 2^{100000}$ and $m$ is sufficiently large. Then the number of permutations $g$ with $v(g)=v \geq \frac{m}{10}$ is at most

$$
\left(\begin{array}{l}
n \\
k
\end{array}\right)^{\frac{1}{2}}(k !)^{\frac{1}{4}} n^{-\frac{m}{800000000}} .
$$

Proof. If $x+y+z \geq \frac{m}{1000}$, then our statement follows from the previous results. Assume otherwise. Suppose first that $k ! \geq n \frac{m}{100}$. The number of choices for small blocks $S_{i}$ with $\left|\operatorname{supp}(g) \cap S_{i}\right|=2$ is at most $\left(\begin{array}{c}n \\ {\left[\frac{k}{2}\right]}\end{array}\right)$. Hence the number of choices for all the pairs $\operatorname{supp}(g) \cap S_{i}$ in these blocks is at most $\left(\begin{array}{c}n \\ {\left[\frac{k}{2}\right]}\end{array}\right)\left(a^{2}\right)^{\left[\frac{k}{2}\right]} \leq\left(\begin{array}{l}n \\ k\end{array}\right)^{\frac{1}{2}}(2 a)^{k}$. The number of choices for $\operatorname{supp}(g)$ is then at most

$$
\left(\begin{array}{l}
n \\
k
\end{array}\right)^{\frac{1}{2}}(2 a)^{k} n^{x+y+z} \leq\left(\begin{array}{l}
n \\
k
\end{array}\right)^{\frac{1}{2}}(2 a)^{k}(k !)^{\frac{1}{10}} .
$$

Hence by Theorem A the number of choices for $g$ itself is at most

$$
\left(\begin{array}{l}
n \\
k
\end{array}\right)^{\frac{1}{2}}(2 a)^{k}(k !)^{\frac{1}{10}} k^{\frac{k}{10000}}
$$

which is less then $\left(\begin{array}{l}n \\ k\end{array}\right)^{\frac{1}{2}}(k !)^{\frac{1}{8}}$ if $m$ is large enough. Therefore in this case the number of permutations $g$ is at most $\left(\begin{array}{l}n \\ k\end{array}\right)^{\frac{1}{2}}(k !)^{\frac{1}{4}} n^{-\frac{m}{800}}$. Suppose now that $k ! \leq n^{\frac{m}{100}}$. To any permutation $g \in G$ we assign a permutation $\bar{g}$ obtained by "forgetting about" $\left[\frac{m}{10}\right]$ transpositions in the small blocks $S_{j}$ of the smallest index $j$ (which $g$ fixes and for which $\left.\left|\operatorname{supp}(g) \cap S_{j}\right|=2\right)$. Note that if $\bar{g}=\bar{h}$ then $\left|\operatorname{supp}\left(g h^{-1}\right)\right| \leq \frac{m}{2}$, hence we have $g=h$. That is, $\bar{g}$ uniquely determines $g$. The number of choices for $\operatorname{supp}(\bar{g})$ is at most

$$
\left(\begin{array}{c}
n \\
{\left[\frac{k}{2}\right]-\left[\frac{m}{10}\right]}
\end{array}\right) a^{k} n^{x+y+z} \leq\left(\begin{array}{l}
n \\
k
\end{array}\right)^{\frac{1}{2}}(2 a)^{k} n^{-\frac{m}{10}} n^{\frac{m}{1000}} .
$$

The number of choices for $\bar{g}$ and hence $g$ is at most

$$
\left(\begin{array}{l}
n \\
k
\end{array}\right)^{\frac{1}{2}}(2 a)^{k} n^{-\frac{m}{10}} n^{\frac{m}{1000}} \cdot k ! \leq\left(\begin{array}{l}
n \\
k
\end{array}\right)^{\frac{1}{2}} n^{-\frac{m}{10}} n^{\frac{m}{1000}}(k !)^{2}
$$


if $m$ is large enough. Hence in this case the number of choices for $g$ is at most

$$
\left(\begin{array}{l}
n \\
k
\end{array}\right)^{\frac{1}{2}} n^{-\frac{m}{10}} n^{\frac{m}{1000}} n^{\frac{m}{50}} \leq\left(\begin{array}{l}
n \\
k
\end{array}\right)^{\frac{1}{2}} \cdot n^{-\frac{m}{20}}
$$

This completes the proof of the proposition.

We need the following auxiliary result.

Lemma 29. Let $H$ be a permutation group of degree $n$ such that each element of order 3 moves at least 100000 points. Assume that $k \leq n^{\frac{2}{3}}$ and $k$ is sufficiently large. Then

$$
\left|H_{k}\right| \leq\left(\begin{array}{l}
n \\
k
\end{array}\right)^{\frac{1}{2}}(k !)^{2} .
$$

Proof. Let $g \in H$ be a permutation with $|\operatorname{supp}(g)|=k$ and $\left|\operatorname{supp}\left(g_{A}\right)\right|=y$. Making use of Corollary 15 we see that the number of choices for $g_{S \cup L}$ is at most $\left(\begin{array}{l}n \\ k\end{array}\right)^{\frac{1}{2}}\left[\frac{y}{2}\right] ! 2^{(a+4) k} n^{-\frac{v}{2}} k !$ which is less than $\frac{1}{k}\left(\begin{array}{l}n \\ k\end{array}\right)^{\frac{1}{2}}(k !)^{2} n^{-\frac{y}{2}}$ if $k$ is large enough. By the remark after Proposition 18 the number of possibilities for $g$ is at most $\frac{1}{k}\left(\begin{array}{l}n \\ k\end{array}\right)^{\frac{1}{2}}(k !)^{2} n^{-\frac{y}{2}} \cdot n \frac{y}{5000} \leq \frac{1}{k}\left(\begin{array}{l}n \\ k\end{array}\right)^{\frac{1}{2}}(k !)^{2}$. Summing over the $k$ ways to chose $y$, our statement follows.

Proposition 30. Assume that $n^{\frac{2}{3}} \geq k \geq 2^{10000}$ and $m$ is sufficiently large. Then the number of permutations $g$ with $v(g)=v \leq \frac{m}{10}$ is at most $\left(\begin{array}{l}n \\ k\end{array}\right)^{\frac{1}{2}}(k !)^{\frac{1}{4}} n^{-\frac{m}{800000000}}$.

Proof. Just like in the proof of Proposition 28 we might assume that $x+y+z \leq \frac{m}{1000}$. Note that in the proof of Proposition 28 we do not use the condition on $v$ in the case $k ! \geq n^{\frac{m}{100}}$, so our statement follows in this case. Now assume that $k ! \leq n^{\frac{m}{100}}$. The number of choices for the $x+y+z+2 v$ points of $\operatorname{supp}(g)$ which are not contained in the two-element blocks moved by $g$ is at most $n^{x+y+z+2 v} \leq n^{\frac{m}{5}+\frac{m}{1000}}$. Let us fix such a set $R$ of $x+y+z+2 v$ points and count the permutations $g$ which correspond to $R$. Denote by $\mathcal{P}$ the set of two-element blocks disjoint from $R$. Each of the permutations $g$ considered induces a permutation $\tilde{g}$ of $\mathcal{P}$ of support $\frac{1}{2}(k-|R|)$. It is clear that $\operatorname{supp}(\tilde{g})$ and $R$ determine $\operatorname{supp}(g)$. Assume first that $k \geq|\mathcal{P}|^{\frac{2}{3}}$. In this case the number of choices for the two-element blocks moved by $\tilde{g}$ is at most $|\mathcal{P}|^{\frac{k}{2}} \leq k^{\frac{3}{4} k} \leq k$ !. Hence the number of choices for $\operatorname{supp}(g)$ is at most $n^{\frac{m}{4}} \cdot k ! \leq n^{\frac{m}{4}+\frac{m}{100}}$. Applying Theorem A, the number of choices for $g$ itself is bounded by $n^{\frac{m}{4}+\frac{m}{100}} k \frac{k}{10000} \leq\left(\begin{array}{l}n \\ k\end{array}\right)^{\frac{1}{2}}$. In this case our statement follows. Assume now that $k \leq|\mathcal{P}|^{\frac{2}{3}}$. Consider the permutation group $\widetilde{G}$ generated by all the 
permutations $\tilde{g}$. We claim that each element of order 3 in $\widetilde{G}$ moves at least $\frac{m}{4}$ points (of $\mathcal{P}$ ). For otherwise let $\tilde{h}$ be an element of order 3 in $\widetilde{G}$ with $|\operatorname{supp}(\tilde{h})| \leq \frac{m}{4}$. Now $\tilde{h}$ can be written as a product $\tilde{h}=\tilde{g}_{1} \ldots \tilde{g}_{t}$ in $\widetilde{G}$ (where the $\tilde{g}_{i}$ are from the above generating set of $\widetilde{G}$, i.e. each $\tilde{g}_{i}$ comes from one of the $g$ ). Consider $h=g_{1} \ldots g_{t} \in$ $G$. It has order divisible by 3 and hence $h^{2}$ is non-trivial. But $h^{2}$ moves only points in $R$ and the points corresponding to the two-element blocks in $\operatorname{supp}(\tilde{h})$. Hence we have $\left|\operatorname{supp}\left(h^{2}\right)\right| \leq \frac{m}{2}+|R|<m$, a contradiction. Applying Lemma 29, we see that the number of possibilities for $\operatorname{supp}(\tilde{g})$ is at most $\left(\begin{array}{c}|\mathcal{P}| \\ {\left[\frac{k}{2}\right]}\end{array}\right)^{\frac{1}{2}}(k !)^{2} \leq\left(\begin{array}{c}n \\ {\left[\frac{k}{2}\right]}\end{array}\right)^{\frac{1}{2}} n^{\frac{m}{50}}$ if $m$ is large enough. Hence the number of choices for $\operatorname{supp}(g)$ is at most

$$
\left(\begin{array}{c}
n \\
{\left[\frac{k}{2}\right]}
\end{array}\right)^{\frac{1}{2}} n^{\frac{m}{5}+\frac{m}{50}+\frac{m}{100}} \leq\left(\begin{array}{c}
n \\
{\left[\frac{k}{2}\right]}
\end{array}\right)^{\frac{1}{2}} n^{\frac{m}{4}} .
$$

The number of choices for $g$ is at most $\left(\begin{array}{c}n \\ {\left[\frac{k}{2}\right]}\end{array}\right)^{\frac{1}{2}} n^{\frac{m}{4}} k ! \leq\left(\begin{array}{c}n \\ {\left[\frac{k}{2}\right]}\end{array}\right)^{\frac{1}{2}} n^{\frac{m}{4}+\frac{m}{100}}$ which implies our statement.

Putting together Lemma 13, Proposition 28 and Proposition 30 we obtain Theorem B.

Acknowledgment. JK acknowledges support by the European Commission under the Integrated Projects RESQ, IST-2001-37559 and Qubit Applications (QAP) funded by the IST directorate as Contract Number 015848, and by ACI Sécurité Informatique SI/03 511 and ANR AlgoQP grants of the French Research Ministry as well as by an Alon Fellowship of the Israeli Council of Academic Research. LP was supported by grants OTKA T049841 and NK 62321. AS wishes to acknowledge an Israeli Science Foundation grant and a Bi-National Science Foundation United States - Israel grant 2004052.

\section{References}

[1] L. Babai, On the order of uniprimitive permutation groups. Ann. of Math. (2) 113 (1981), 553-568. Zbl 0485.20002 MR 621016

[2] L. Babai, On the order of doubly transitive permutation groups. Invent. Math. 65 (1982), 473-484. Zbl 0478.20002 MR 643565

[3] R. Beals, Quantum computation of Fourier transforms over symmetric groups. In STOC '97 (El Paso, TX, 1997), ACM, New York 1999, 48-53. Zbl 0962.68070 MR 1715623

[4] P. J. Cameron, Finite permutation groups and finite simple groups. Bull. London Math. Soc. 13 (1981), 1-22. Zbl 0463.20003 MR 599634 
[5] J. D. Dixon and B. Mortimer, Permutation groups. Grad. Texts in Math. 163, SpringerVerlag, New York 1996. Zbl 0951.20001 MR 1409812

[6] M. Ettinger and P. Høyer, On quantum algorithms for noncommutative hidden subgroups. Adv. in Appl. Math. 25 (2000), 239-251. Zbl 0967.68076 MR 1783552

[7] K. Friedl, G. Ivanyos, F. Magniez, M. Santha, and P. Sen, Hidden translation and orbit coset in quantum computing. In STOC 2003 (San Diego, CA, 2003), ACM, New York 2003, 1-9. Zbl 1074.68503 MR 2121067

[8] V. D. Goppa, A new class of linear error-correcting codes. Problemy Peredači Informacii 6 (1970), 24-30 (in Russian). Zbl 0292.94011 MR 0294040

[9] D. Gorenstein, Classifying the finite simple groups. Bull. Amer. Math. Soc. (N.S.) 14 (1986), 1-98. Zbl 0585.20003 MR 818060

[10] R. L. Graham, M. Grötschel, and L. Lovasz (ed.), Handbook of combinatorics. Elsevier, Amsterdam; MIT Press, Cambridge, MA, 1995. Zbl 0833.05001 MR 1373655

[11] M. Grigni, L. Schulman, M. Vazirani, and U. Vazirani, Quantum mechanical algorithms for the nonabelian hidden subgroup problem. In STOC 2001 (Hersonissos, 2001, Greece), ACM, New York 2001, 68-74. Zbl 1074.68500 MR 2120302

[12] R. Guralnick and K. Magaard, On the minimal degree of a primitive permutation group. J. Algebra 207 (1998), 127-145. Zbl 0911.20003 MR 1643074

[13] S. Hallgren, C. Moore, M. Rötteler, A. Russell, and P. Sen, Limitations of quantum coset states for graph isomorphism. In STOC'06 (Seattle, WA, 2006) , ACM, New York 2006, 604-617. Zbl 05134547 MR 2277186

[14] S. Hallgren, A. Russell, and A. Ta-Shma, Normal subgroup reconstruction and quantum computation using group representations. In STOC 2000 (Portland, Oregon, 2000), ACM, New York 2000, 627-635. Zbl 1074.68501 MR 2115301

[15] G. Ivanyos, F. Magniez, and M. Santha, Efficient quantum algorithms for some instances of the non-abelian hidden subgroup problem. Internat. J. Found. Comput. Sci. 14 (2003), 723-739. Zbl 1101.68582 MR 2022781

[16] C. Jordan, Sur la limite de transitivité des groupes non alternés. Bull. Soc. Math. France 1 (1872/73), 40-71. JFM 07.0073.02 MR 1503635

[17] C. Jordan, Sur la limite de degré des groupes primitifs qui contiennent une substitution donnée. J. Reine Angew. Math. 79 (1875), 248-258. JFM 06.0081.02

[18] J. Kempe and A. Shalev, The hidden subgroup problem and permutation group theory. In SODA '05 (Vancouver, BC, 2005), ACM, New York 2005, 1118-1125. Zbl 1073.68886 MR 2298372

[19] L. G. Kovács and G. R. Robinson, On the number of conjugacy classes of a finite group. J. Algebra 160 (1993), 441-460. Zbl 0830.20048 MR 1244923

[20] G. Kuperberg, A subexponential-time quantum algorithm for the dihedral hidden subgroup problem. SIAM J. Comput. 35 (2005), 170-188. Zbl 1084.81019 MR 2178804

[21] F. Levy-dit-Vehel and S. Litsyn, Parameters of Goppa codes revisited. IEEE Trans. Inform. Theory 43 (1997), 1811-1819. Zbl 1053.94562 MR 1481040 
[22] M.W. Liebeck, Bounds for the orders of some transitive permutation groups. Bull. London Math. Soc. 14 (1982), 337-344. Zbl 0467.20003 MR 663484

[23] M. W. Liebeck, On the orders of transitive permutation groups. Bull. London Math. Soc. 16 (1984), 523-524. Zbl 0551.20001 MR 751825

[24] M. W. Liebeck and J. Saxl, Minimal degrees of primitive permutation groups, with an application to monodromy groups of covers of Riemann surfaces. Proc. London Math. Soc. (3) 63 (1991), 266-314. Zbl 0696.20004 MR 1114511

[25] M. W. Liebeck and A. Shalev, Diameters of finite simple groups: sharp bounds and applications. Ann. of Math. (2) 154 (2001), 383-406. Zbl 1003.20014 MR 1865975

[26] F. MacWilliams and N. J. A. Sloane, The theory of error-correcting codes. North-Holland Math. Library 16, North-Holland, Amsterdam 1977. Zbl 0369.94008 MR 0465509

[27] C. Moore, D. Rockmore, and A. Russell, Generic quantum Fourier transforms. ACM Trans. Algorithms 2 (2006), 707-723. MR 2284252

[28] C. Moore, D. Rockmore, A. Russell, and L. J. Schulman, The power of basis selection in Fourier sampling: hidden subgroup problems in affine groups. In SODA '04 (New Orleans, LA, 2004), ACM, New York 2004, 1113-1122. Zbl 1076.68606 MR 2291004

[29] A. R. C. Moore and L. Schulman, The symmetric group defies strong Fourier sampling. In FOCS '05 (Pittsburgh, PA, 2005), IEEE Computer Society, 2005, 479-490.

[30] M. A. Nielsen and I. L. Chuang, Quantum computation and quantum information. Cambridge University Press, Cambridge 2000. Zbl 1049.81015 MR 1796805

[31] C. E. Praeger and J. Saxl, On the orders of primitive permutation groups. Bull. London Math. Soc. 12 (1980), 303-307. Zbl 0443.20001 MR 576980

[32] O. Regev, A subexponential time algorithm for the dihedral hidden subgroup problem with polynomial space. Technical report, 2004. arXiv:quant-ph/0406151

[33] M. Rötteler and T. Beth, Polynomial-time solution to the hidden subgroup problem for a class of non-abelian groups. Technical report, 1998. arXiv:quant-ph/9812070.

[34] B. E. Sagan, The symmetric group. Grad. Texts in Math. 203, Springer-Verlag, New York 2001 Zbl 0964.05070 MR 1824028

[35] P. W. Shor, Polynomial-time algorithms for prime factorization and discrete logarithms on a quantum computer. SIAM J. Comput. 26 (1997), 1484-1509. Zbl 1005.11065 MR 1471990

Received December 26, 2006

J. Kempe, School of Computer Science, Tel Aviv University, Tel Aviv 69978, Israel, and CNRS \& LRI, Université de Paris-Sud, 91405 Orsay Cedex, France

E-mail: kempe@post.tau.ac.il

L. Pyber, Mathematical Institute of the Hungarian Academy of Sciences, P.O. Box 127, 1364 Budapest, Hungary

E-mail: pyber@renyi.hu

A. Shalev, Institute of Mathematics, The Hebrew University, Jerusalem 91904, Israel

E-mail: shalev@math.huji.ac.il 